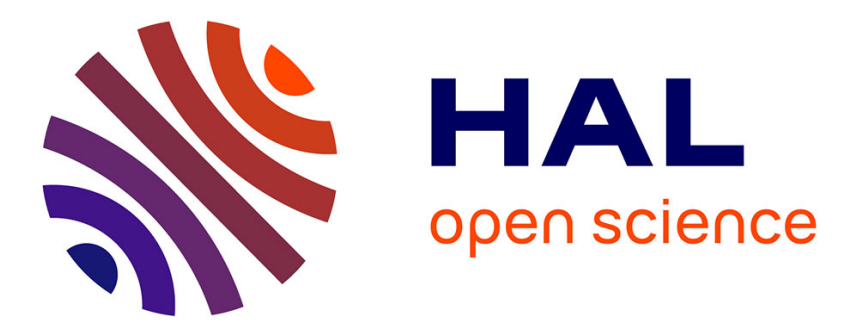

\title{
Hamiltonian fluid reductions of electromagnetic drift-kinetic equations for an arbitrary number of moments \\ E Tassi
}

\section{To cite this version:}

E Tassi. Hamiltonian fluid reductions of electromagnetic drift-kinetic equations for an arbitrary number of moments. Annals of Physics, 2015, 362, pp.239. 10.1016/j.aop.2015.08.001 . hal-01188469

\section{HAL Id: hal-01188469 \\ https://hal.science/hal-01188469}

Submitted on 30 Aug 2015

HAL is a multi-disciplinary open access archive for the deposit and dissemination of scientific research documents, whether they are published or not. The documents may come from teaching and research institutions in France or abroad, or from public or private research centers.
L'archive ouverte pluridisciplinaire HAL, est destinée au dépôt et à la diffusion de documents scientifiques de niveau recherche, publiés ou non, émanant des établissements d'enseignement et de recherche français ou étrangers, des laboratoires publics ou privés. 


\title{
Hamiltonian fluid reductions of electromagnetic drift-kinetic equations for an arbitrary number of moments
}

\author{
E. Tassi ${ }^{1}$ \\ ${ }^{1}$ Aix-Marseille Université, Université de Toulon, CNRS, Centre de Physique Théorique, \\ UMR 7332, 13288 Marseille, France
}

\begin{abstract}
We present an infinite family of Hamiltonian electromagnetic fluid models for plasmas, derived from drift-kinetic equations. An infinite hierarchy of fluid equations is obtained from a Hamiltonian drift-kinetic systems by taking moments of a generalized distribution function and using Hermite polynomials as weight functions of the velocity coordinate along the magnetic guide field. Each fluid model is then obtained by truncating the hierarchy to a finite number $N+1$ of equations by means of a closure relation. We show that, for any positive $N$, a linear closure relation between the moment of order $N+1$ and the moment of order $N$ guarantees that the resulting fluid model possesses a Hamiltonian structure, thus respecting the Hamiltonian character of the parent drift-kinetic model. An orthogonal transformation is identified which maps the fluid moments to a new set of dynamical variables in terms of which the Poisson brackets of the fluid models become a direct sum and which unveils remarkable dynamical properties of the models in the two-
\end{abstract}


dimensional (2D) limit. Indeed, when imposing translational symmetry with respect to the direction of the magnetic guide field, all models belonging to the infinite family can be reformulated as systems of advection equations for Lagrangian invariants transported by incompressible generalized velocities. These are reminiscent of the advection properties of the parent drift-kinetic model in the 2D limit and are related to the Casimirs of the Poisson brackets of the fluid models. The Hamiltonian structure of the generic fluid model belonging to the infinite family is illustrated treating a specific example of a fluid model retaining five moments in the electron dynamics and two in the ion dynamics. We also clarify the connection existing between the fluid models of this infinite family and some fluid models already present in the literature.

Keywords: Drift-kinetic equations, Noncanonical Poisson brackets, Fluid closures, Reduced fluid models for plasmas

\section{Introduction}

Fluid models represent a commonly adopted and effective tool for modeling both astrophysical and laboratory plasmas. In particular, they are appealing because of the relatively limited amount of computational resources they require, when compared to kinetic models, in order to perform numerical simulations. On the other hand, kinetic models are superior in the range of phenomena they can describe, one example being physical events involving wave-particle interactions, which go beyond the capabilities of purely fluid models and which require a kinetic description instead. Starting from these facts, in the plasma physics community an important effort has been made in order to derive refined fluid models offering a good compromise between the computational effectiveness of a fluid model, evolving a small number of fluid moments, and the accuracy of a kinetic theory. Fluid models are typically derived by taking moments of kinetic equations, and truncating the 
resulting infinite hierarchy of fluid equations at some order $N$, by imposing a closure relation through which the moment of order $N+1$ is expressed in terms of lower order moments. Therefore, in the effort of deriving a refined fluid model, crucial roles are played by the number of moments involved and by the adopted closure relation. In the plasma physics literature, different important criteria for closing a fluid hierarchy have been presented. For instance, an active line of research over the years has concerned the derivation of closures that retain kinetic effects such as Landau damping, or in general that lead to fluid models exhibiting agreement with the parent kinetic model at the level of the linear theory. This has led to the formulation of sophisticated fluid and gyrofluid models retaining kinetic effects for the description of electrostatic instabilities [1], turbulence [2,3,4] and mirror-trapped particles [5]. Electromagnetic Landau fluid models have been presented in Refs. [6, 7] and magnetohydrodynamic models with Landau closures have been derived in Refs. $[8,9,10]$. In other approaches, emphasis has been put on closures guaranteeing an energy conservation theorem [11, 12]. A non-dissipative closure respecting time reversal symmetry of collisionless kinetic equations has been derived in Ref. [13]. In all the above mentioned models, the number of fluid moments retained for each particle species never goes beyond four, which corresponds to accounting at most for heat fluxes dynamics. On the other hand, fluid truncations are also used to solve kinetic equations numerically, often in one dimension in velocity space, a recent example, pertinent in particular to the present paper, being provided by Ref. [14]. In these cases, it is necessary to retain a very large number of moments $N \gg 1$, in order to have an accurate description of the kinetic dynamics.

An approach which is complementary to that typically followed in the plasma physics community consists of examining the problem of closure of fluid plasma models from the point of view of the structure of the resulting equations when seen as an infinite-dimensional dynamical system. In particular, if the parent kinetic system possesses a Hamiltonian structure (which is the case for the paradigmatic Vlasov-Maxwell system $[15,16]$, and should be the case, in general, for collisionless non-dissipative drift and gyrokinetic models ) it would be desirable that the adopted closure preserves such structure, or, in general, that it permits to identify dissipative terms in the fluid model, with the constraint that, if such terms are removed, the resulting fluid model be Hamiltonian. In fact, the introduction of uncontrolled dissipation in the system could not only violate energy conservation but also other conservation laws related to Casimir invariants, which are characteristic of fluid 
models formulated in terms of Eulerian variables [17]. Also, the knowledge of the Hamiltonian structure of a fluid model in its non-dissipative limit, can be used for stability analyses $[17,18]$ or also for structure preserving finitemode approximations $[19,20]$. The relation between the Poisson bracket of the Vlasov equation and that of the infinite hierarchy of fluid equations obtained by taking moments had been investigated in Ref. [21], where it was shown that the operation of taking moments is a Poisson map and that the resulting bracket is the one presented in Ref. [22]. More recently, the relation between the truncated moment hierarchy of the geodesic Vlasov equation and integrable systems has been elucidated in Ref. [23]. A geometric interpretation of the Lie-Poisson structure associated with the dynamics of moments has been provided in Ref. [24]. In the context of the studies of the Hamiltonian structure of models obtained by taking moments, we mention also the derivation of the Lie-Poisson structure of the Bogoliubov-Born-GreenKirkwood-Yvon hierarchy in Ref. [25]. A considerable amount of activity, on the other hand, has concerned finite reductions of hydrodynamic chains (see, for instance Ref. [26] for a recent application, and references therein).

With regard to the specific problem of determining closures that respect the Hamiltonian structure of the parent model, we mention that in the case of the Vlasov system, subalgebras for the Vlasov-Poisson bracket expressed in terms of the fluid moments, have been identified in Ref. [27]. Recent results, on the other hand, concern the identification of closure relations that, when inserted into the bracket of the fluid moments, truncate it in such a way that the resulting bracket is still a Poisson bracket. In the case when the parent model is a drift-kinetic one, it has been shown that the adiabatic closure is the only one possessing this property [28, 29]. Linear closures, on the other hand, are those selected for drift and gyrokinetic models in the " $\delta f$ approximation" $[28,30]$. Following this approach also a new Hamiltonian fluid model obtained from the Vlasov equation has been derived [31]. These results, however, concern fluid models retaining only a very low number of moments, more precisely two-moment models for Refs. [28, 29, 30] and threemoment models for Ref. [31].

In this paper we present a closure relation which yields electromagnetic Hamiltonian fluid models, from a Hamiltonian drift-kinetic model, for an arbitrary number of moments. The parent drift-kinetic model is based on the " $\delta f$ approximation" and is formulated adopting, as dynamical variable, a generalized distribution function $g_{e}$, depending on the actual perturbation of the electron distribution function and on the magnetic potential. Moments 
are taken with respect to $g_{e}$ using Hermite polynomials as weight functions. The Hamiltonian structure of the resulting fluid model is derived in the case in which the parent model is a hybrid model where the electron species is described by a drift-kinetic equation, whereas the ions are treated with a gyrofluid description. However, the procedure can easily be extended to a purely drift-kinetic Hamiltonian parent model with an arbitrary number of species. The derivation of the Hamiltonian structure unveils also a hidden structure in the resulting fluid models, which becomes remarkable in the 2D limit, when translational symmetry along the direction of the magnetic guide field is imposed. In this limit, indeed the dynamics of the models can be reformulated entirely in terms of Lagrangian invariants advected by generalized incompressible velocity fields. The presence of such invariants is related to the existence of Casimirs for the system, which in turn are associated with the Poisson bracket. It turns out that some of the above mentioned fluid models presented in the literature, in some specific limit, possess such structure.

Clearly, the drift-kinetic system included in the parent model, and the descendant fluid equations, are highly simplified models and are not meant to provide a precise quantitive description of a specific experimental situation. In particular, the models suffer from limitations due to the simplified slab geometry (implying the absence of curvature effects) and to the suppression of finite Larmor radius effects. With regard to this, some results concerning Hamiltonian closures for fluid models obtained from a gyrokinetic, instead of a drift-kinetic system, have been described in Ref. [30]. The adopted $\delta f$ approximation, also imposes a further restriction, by limiting the analysis to plasmas close to a Maxwellian equilibrium with uniform temperature. Extensions to bi-Maxwellian equilibria or to equilibria with temperature gradients are under development. Finally, because we are restricting to the Hamiltonian portion of a model, all damping effects due to collisions or to kinetic effects possibily retained in the fluid models, are not taken into account. The utility of the adopted models, on the other hand, lies also in their relative simplicity, which makes them amenable to an analytical treatment and permits to extract leading order information about plasma phenomena. For instance, fluid models such as those derived in this manuscript, have been used to investigate main basic features of collisionless magnetic reconnection $[32,33,34]$.

The paper is organized as follows. In Sec. 2 the hybrid parent model is introduced and its Hamiltonian structure is presented. In Sec. 3, after 
reviewing a few basic properties of Hermite polynomials, we present the infinite hierarchy of fluid equations and introduce the closure relation. In Sec. 4, the Hamiltonian structure of the fluid models is formulated and analyzed, with emphasis on conservation laws and connections with models existing in the literature. The Hamiltonian structure derived in general is also exemplified treating the case of a $5+2$ field model. Sec. 5 is devoted to a discussion of the results and to conclusions.

\section{Hamiltonian parent model}

In a Cartesian system $(x, y, z)$ for the spatial coordinates we consider the following drift-kinetic evolution equation for the electron species, in the so called " $\delta f$ approximation":

$$
\frac{\partial g_{e}}{\partial t}+\frac{c}{B}\left[\phi-\frac{v}{c} A, g_{e}\right]+v \frac{\partial}{\partial z}\left(g_{e}-e \frac{\mathcal{F}_{e}}{T_{e}}\left(\phi-\frac{v}{c} A\right)\right)=0,
$$

complemented by the quasi-neutrality relation

$$
\frac{e^{2}}{T_{i}} n_{0}\left(1-\Gamma_{0}\right) \phi=-e \Gamma_{0}^{1 / 2} n_{i}+e \int d v g_{e}
$$

and by Ampère's law

$$
\frac{c}{4 \pi} \Delta A-\frac{e^{2} n_{0}}{m_{e} c} A=-e n_{0} \Gamma_{0}^{1 / 2} u_{i}+e \int d v v g_{e}
$$

The evolution of the ions, on the other hand, is assumed to be governed by the continuity equation

$$
\frac{\partial n_{i}}{\partial t}+\frac{c}{B}\left[\Gamma_{0}^{1 / 2} \phi, n_{i}\right]-\frac{n_{0}}{B}\left[\Gamma_{0}^{1 / 2} A, u_{i}\right]+n_{0} \frac{\partial u_{i}}{\partial z}=0
$$

and by the momentum equation

$$
\frac{\partial D}{\partial t}+\frac{c}{B}\left[\Gamma_{0}^{1 / 2} \phi, D\right]-\frac{T_{i}}{M B}\left[\Gamma_{0}^{1 / 2} A, n_{i}\right]+\frac{T_{i}}{M} \frac{\partial n_{i}}{\partial z}+\frac{e n_{0}}{M} \frac{\partial \Gamma_{0}^{1 / 2} \phi}{\partial z}=0
$$

In Eq. (1), the function $g_{e}$ is defined as

$$
g_{e}(x, y, z, v, t)=\tilde{f}_{e}(x, y, z, v, t)-\frac{e}{T_{e}} \frac{v}{c} \mathcal{F}_{e}(v) A(x, y, z, t),
$$


where $\tilde{f}_{e}$ is the perturbation of the electron distribution function averaged over the magnetic moment with respect to the equilibrium Maxwellian

$$
\mathcal{F}_{e}(v)=n_{0} \sqrt{\frac{m_{e}}{2 \pi T_{e}}} \mathrm{e}^{-m_{e} v^{2} /\left(2 T_{e}\right)},
$$

with $v$ indicating the velocity coordinate along $z$, which is the direction of the magnetic guide field, and with the constants $e, c, m_{e}, n_{0}$ and $T_{e}$ indicating the proton charge, the speed of light, the electron mass and the equilibrium density and temperature for the electrons, respectively. We remark that $\tilde{f}_{e}$ and $\mathcal{F}_{e}$ correspond to averages of the actual perturbation and equilibrium distribution functions, over the magnetic moment. More precisely $\tilde{f}_{e}(x, y, z, v, t)=$ $\left(2 \pi B / m_{e}\right) \int d \mu \mathrm{f}_{e}(x, y, z, v, \mu, t)$ and $\mathcal{F}_{e}(v)=\left(2 \pi B / m_{e}\right) \int d \mu \mathrm{F}_{e}(v, \mu)$, with $\mu$ indicating the electron magnetic moment, $\mathrm{f}_{e}$ the actual perturbation of the drift-kinetic distribution function and $\mathrm{F}_{e}(v, \mu)=n_{0}\left(2 \pi T_{e} / m_{e}\right)^{-3 / 2} \exp \left(-m_{e} v^{2} / 2 T_{e}-\mu B / T_{e}\right)$. For a straight and homogeneous background magnetic field and in the absence of finite Larmor radius effects, it is indeed possible to obtain the equation (1) for $\tilde{f}_{e}$, independent on $\mu$, by averaging the actual " $\delta f$ " drift-kinetic equation for $\mathrm{f}_{e}$, which can be found, for instance in Ref. [12] and which, under the above assumptions, reads

$$
\frac{\partial \mathrm{g}_{e}}{\partial t}+\frac{c}{B}\left[\phi-\frac{v}{c} A, \mathrm{~g}_{e}\right]+v \frac{\partial}{\partial z}\left(\mathrm{~g}_{e}-e \frac{\mathrm{F}_{e}}{T_{e}}\left(\phi-\frac{v}{c} A\right)\right)=0 .
$$

In Eq. (8) we set $\mathrm{g}_{e}=\mathrm{f}_{e}-\left(e / T_{e}\right)(v / c) \mathrm{F}_{e} A$. Because in Eq. (8) the coefficients do not depend on $\mu$, the average over the magnetic moment leads immediately to Eq. (1). Note also that we use $g_{e}$ as dynamical variable, instead of $h_{e}=\tilde{f}_{e}-e \phi / T_{e}$, which is commonly adopted in drift-kinetic descriptions. The use of $g_{e}$ indeed turns out to be more natural from the point of view of the Hamiltonian formulation of the model.

In our setting the magnetic field is given by $\mathbf{B}=\nabla A \times \hat{z}+B \hat{z}$, with constant $B$, whereas $\phi$ indicates the electrostatic potential. The ion mass and equilibrium temperature are denoted with $M$ and $T_{i}$, respectively. The parallel ion canonical momentum per unit mass $D$ is defined as $D=n_{0}\left(u_{i}+\right.$ $\left.(e /(M c)) \Gamma_{0}^{1 / 2} A\right)$ whereas $n_{i}$ and $u_{i}$ represent the ion guiding center density and parallel velocity perturbations. The operator $\Gamma_{0}^{1 / 2}$ is related to the operator $\Gamma_{0}$ [5], which corresponds, in Fourier space, to the multiplication times the function $I_{0}\left(k_{\perp}^{2} \rho_{i}^{2}\right) \mathrm{e}^{-k_{\perp}^{2} \rho_{i}^{2}}$, with $I_{0}$ indicating the zero order modified Bessel function, $k_{\perp}^{2}=k_{x}^{2}+k_{y}^{2}$ the squared perpendicular wave vector 
and $\rho_{i}=\sqrt{T_{i} / M} M c /(e B)$ the thermal ion gyro radius. Finally, we indicated with the symbol $\Delta$ the Laplacian operator, whereas the bracket [, ] is defined as $[f, g]=\partial_{x} f \partial_{y} g-\partial_{y} f \partial_{x} g$, for two functions $f$ and $g$. We assume that the fields $n_{i}, u_{i}, A$ and $\phi$ are defined on a bounded domain $\mathcal{D} \subset \mathbb{R}^{3}$, over which they are periodic and with zero mean values. The distribution function $\tilde{f}_{e}$ is defined over $\mathcal{D} \times \mathbb{R}$ and is assumed to satisfy periodic boundary conditions over $\mathcal{D}$ and to decay to zero sufficiently fast for $v \rightarrow \infty$.

In order to simplify the exposition of the results, we specialize here to the case in which, in the parent model, only the electrons follow a driftkinetic description, whereas the ions follow a gyro-fluid description with an isothermal closure (Eqs. (4) and (5)) and quasi-neutrality is imposed in the form (2). However, as anticipated in Sec. 1, we remark that the results presented in this paper, can easily be extended to purely drift-kinetic models with multiple species, provided that the drift-kinetic equations are of the form (1) and that the corresponding Poisson's equation and Ampère's law belong to the general form

$$
\phi=L_{0}{ }^{-1} \sum_{j=1}^{\mathcal{N}} q_{j} \int d v g_{j}, \quad A=L_{1}^{-1} \sum_{j=1}^{\mathcal{N}} q_{j} \int d v v g_{j}
$$

respectively. In Eq. (9) we considered $\mathcal{N}$ drift-kinetic species. The evolution of the $j$ th species is described in terms of a function $g_{j}=\tilde{f}_{j}+\left(q_{j} v /\left(T_{j} c\right)\right) \mathcal{F}_{j} A$ defined analogously to $(6)$, where $q_{j}, T_{j}$ and $\mathcal{F}_{j}$ are the charge, the equilibrium temperature and the equilibrium Maxwellian of the species under consideration. The expressions $L_{0}$ and $L_{1}$ in Eq. (9) indicate linear operators that, for the sake of generality, we left unspecified, but that we require to be linear, invertible independent on the coordinate $v$, and symmetric (i.e. formally self-adjoint) with respect to the inner product in $L^{2}(\mathcal{D})$, so that $\int_{\mathcal{D}} d^{3} x f L_{0}{ }^{-1} g=\int_{\mathcal{D}} d^{3} x g L_{0}{ }^{-1} f$ and $\int_{\mathcal{D}} d^{3} x f L_{1}{ }^{-1} g=\int_{\mathcal{D}} d^{3} x g L_{1}{ }^{-1} f$ for functions $f$ and $g$ defined on $\mathcal{D}$. With similar assumptions, the presence of multiple fluid (or gyrofluid) species can also be easily incorporated in the initial model. The choice of a hybrid parent model with drift-kinetic electrons and fluid ions could, however, have an interest of its own, for instance in the context of collisionless magnetic reconnection studies [35].

For a derivation of the parent model from first principles one can refer, for instance to Ref. [12]. Concerning the electron drift-kinetic equations we have here considered the particular case of a straight magnetic field and neglected finite Larmor effects. The ion equations (4)-(5), on the other hand, corre- 
spond to the equations that can be obtained from the gyrokinetic extension of the drift-kinetic model, by imposing an isothermal closure.

In order to derive a Hamiltonian fluid model from a parent model, we require also the latter to possess a Hamiltonian structure in its non-dissipative limit. The parent model under consideration is composed by the evolution equations (1), (4), (5) and by the relations (2)-(3). This model can indeed be cast in the Hamiltonian form

$$
\frac{\partial \chi_{i}}{\partial t}=\left\{\chi_{i}, H\right\}, \quad i=1,2,3
$$

where $\chi_{1}=g_{e}, \chi_{2}=n_{i}$ and $\chi_{3}=D$. Because in a field theory the Poisson bracket acts on functionals, in Eq. (10) $\chi_{i}$ has to be intended as functional, i.e. $\chi_{i}(x)=\int d^{3} x^{\prime} \delta\left(x^{\prime}-x\right) \chi_{i}\left(x^{\prime}\right)$. The Hamiltonian functional $H$ is given by

$$
H=\mathcal{H}_{e}+\mathcal{H}_{i}+\mathcal{H}_{c}
$$

where

$$
\begin{array}{r}
\mathcal{H}_{e}=\frac{1}{2} \int d^{3} x d v \frac{T_{e}}{\mathcal{F}_{e}} g_{e}^{2}, \\
\mathcal{H}_{i}=\frac{1}{2} \int d^{3} x\left(\frac{T_{i}}{n_{0}} n_{i}^{2}+\frac{M}{n_{0}} D^{2}\right), \\
\mathcal{H}_{c}=-\frac{e}{2} \int d^{3} x \phi\left(\int d v g_{e}-\Gamma_{0}^{1 / 2} n_{i}\right) \\
+\frac{e}{2 c} \int d^{3} x A\left(\int d v v g_{e}-\Gamma_{0}^{1 / 2} D\right) .
\end{array}
$$

The symbol $\{$,$\} in Eq. (10) indicates a Poisson bracket, that is an anti-$ symmetric bilinear operation satisfying the Leibniz and Jacobi identity. For our parent hybrid model the Poisson bracket is of noncanonical type and its expression corresponds to

$$
\{F, G\}=\{F, G\}_{e}+\{F, G\}_{i}
$$

where

$$
\begin{aligned}
\{F, G\}_{e} & =\int d^{3} x d v\left(\frac{c}{e B} g_{e}\left[F_{g_{e}}, G_{g_{e}}\right]-v \frac{\mathcal{F}_{e}}{T_{e}} F_{g_{e}} \frac{\partial G_{g_{e}}}{\partial z}\right), \\
\{F, G\}_{i} & =-\frac{c}{e B} \int d^{3} x\left(n_{i}\left[F_{n_{i}}, G_{n_{i}}\right]+D\left(\left[F_{n_{i}}, G_{D}\right]+\left[F_{D}, G_{n_{i}}\right]\right)+\frac{T_{i}}{M} n_{i}\left[F_{D}, G_{D}\right]\right) \\
& -\frac{n_{0}}{M} \int d^{3} x\left(F_{D} \frac{\partial G_{n_{i}}}{\partial z}+F_{n_{i}} \frac{\partial G_{D}}{\partial z}\right),
\end{aligned}
$$


with subscripts on functionals indicating functional derivatives. In order to derive Eqs. (1), (4) and (5) from the Hamiltonian (11) and the bracket (15) use must be made of the relations

$$
H_{g_{e}}=\frac{T_{e}}{\mathcal{F}_{e}} g_{e}-e\left(\phi-\frac{v}{c} A\right), \quad H_{n_{i}}=e \Gamma_{0}^{1 / 2} \phi+\frac{T_{i}}{n_{0}} n_{i}, \quad H_{D}=M u_{i} .
$$

These relations can be obtained from Eq. (11) and considering that, from Eqs. (2)-(3), the electrostatic and magnetic potentials can be expressed as

$$
\phi=L_{0}^{-1} e\left(\Gamma_{0}^{1 / 2} n_{i}-\int d v g_{e}\right), \quad A=L_{1}^{-1} e\left(\Gamma_{0}^{1 / 2} D-\int d v v g_{e}\right)
$$

where $L_{0,1}$ are operators with properties analogous to those of Eq. (9). In this specific case, explicit expressions for $L_{0,1}$ can be obtained in Fourier space, assuming zero mean value for $\phi$, by inverting the relations (2)-(3) and recalling that $n_{0} \Gamma_{0}^{1 / 2} u_{i}=\Gamma_{0}^{1 / 2} D-\left(n_{0} e / M c\right)\left(\Gamma_{0}^{1 / 2}\right)^{2} A$. In particular, for the symmetric operators $\Gamma_{0}$ and $\Gamma_{0}^{1 / 2}$ in Fourier space, the commonly adopted Padé approximations $\Gamma_{0} \approx\left(1+k_{\perp}^{2} \rho_{i}^{2}\right)^{-1}$ and $\Gamma_{0}^{1 / 2} \approx\left(1+k_{\perp}^{2} \rho_{i}^{2} / 2\right)^{-1}$ can be used.

We remark that the Poisson bracket (15) is given by the direct sum of two independent Poisson brackets: the first one, corresponding to $\{F, G\}_{e}$, concerns the electron drift-kinetic species, whereas $\{F, G\}_{i}$ involves the ion quantities. These two brackets have appeared independently in different contexts as Poisson brackets for drift-and gyrokinetic [36, 28] models and for reduced fluid models [37], respectively. The coupling between the electron and ion dynamics, on the other hand, is provided through the Hamiltonian, and in particular via the term $\mathcal{H}_{c}$, which involves the electrostatic energy and part of the magnetic energy.

\section{Hierarchy of fluid equations and closure}

In this section we introduce the infinite hierarchy of fluid equations and the closure that we adopt to truncate the hierarchy. Before proceeding with the derivation of the fluid model, we find it appropriate to recall a few basic properties about Hermite polynomials, which are the weight functions that will be used in the operation of taking moments of the generalized distribution function $g_{e}$. First, we recall that the use of the Hermite decomposition for kinetic distribution functions, and in particular for the Vlasov distribution function, begins some decades ago $[38,39,40]$. More recent applications 
to kinetic plasma theories have concerned, for instance, the derivation of gyrofluid models for plasma turbulence [3, 12], fluid models for ion temperature gradient instabilities [13], as well as hybrid models for collisionless reconnection $[41,14]$.

After denoting with $v_{t e}=\sqrt{T_{e} / m_{e}}$ the equilibrium electron thermal speed, we indicate with $H_{m}\left(v / v_{t e}\right)$ the $m$ th Hermite polynomial, defined as

$$
H_{m}\left(\frac{v}{v_{t e}}\right)=(-1)^{m} \mathrm{e}^{\frac{v^{2}}{2 v_{t e}^{2}}} \frac{d^{m}}{d\left(\frac{v}{v_{t e}}\right)^{m}} \mathrm{e}^{-\frac{v^{2}}{2 v_{t e}^{2}}}
$$

for any non-negative integer $m$. From the definition (20) it follows that the first Hermite polynomials are given by $H_{0}=1, H_{1}=v / v_{t e}, H_{2}=\left(v / v_{t e}\right)^{2}-1$, $H_{3}=\left(v / v_{t e}\right)^{3}-3\left(v / v_{t e}\right), \cdots$.

Hermite polynomials satisfy the following orthogonality relation:

$$
\int d v H_{m}\left(\frac{v}{v_{t e}}\right) H_{n}\left(\frac{v}{v_{t e}}\right) \mathcal{F}_{e}(v)=n_{0} n ! \delta_{n m} .
$$

Hermite polynomials also form a complete set over $-\infty \leq v \leq \infty$. Therefore they can be used to decompose the function $g_{e}$ in the following way:

$$
g_{e}(x, y, z, v, t)=\sum_{n=0}^{+\infty} \frac{g_{n}(x, y, z, t)}{\sqrt{n !}} H_{n}\left(\frac{v}{v_{t e}}\right) \mathcal{F}_{e}(v)
$$

The coefficients $g_{n}$, by virtue of Eq. (21), can be obtained from $g_{e}$ making use of the relation

$$
g_{n}=\frac{1}{n_{0} \sqrt{n !}} \int d v H_{n} g_{e} .
$$

Because each Hermite polynomial is a linear combination of powers of $v / v_{t e}$, from Eq. (23) it follows that the coefficients $g_{n}$ are linear combinations of the fluid moments $\int d v\left(v / v_{t e}\right)^{n} g_{e}$ of $g_{e}$, with $n$ a non-negative integer. It is then straightforward to perform the change of coordinates from the moments in terms of Hermite polynomials to the ordinary moments and viceversa, if needed.

The coefficients $g_{n}$ will be the dynamical variables of the fluid models that we will derive. We remark that the lowest order coefficients have a direct physical interpretation in terms of commonly adopted fluid variables, in dimensionless form. Indeed one has 


$$
\begin{aligned}
& g_{0}=\frac{n_{e}}{n_{0}}, \quad g_{1}=\frac{u_{e}}{v_{t e}}-\frac{e}{m_{e} v_{t e} c} A, \\
& g_{2}=\frac{T_{e \|}}{\sqrt{2} m_{e} v_{t e}^{2}}, \quad g_{3}=\sqrt{\frac{2}{3}} \frac{q_{e \|}}{n_{0} T_{e} v_{t e}},
\end{aligned}
$$

where $n_{e}=\int d v \tilde{f}_{e}, u_{e}=\int d v v \tilde{f}_{e} / n_{0}, T_{e \|}=\left(m_{e} v_{t e}^{2} / n_{0}\right) \int d v\left(v^{2} / v_{t e}^{2}-1\right) \tilde{f}_{e}$, $q_{e \|}=\left(m_{e} v_{t e}^{3} / 2\right) \int d v\left(v^{3} / v_{t e}^{3}-3 v / v_{t e}\right) \tilde{f}_{e}$. Therefore, $g_{0}, g_{1}, g_{2}$ and $g_{3}$ represent electron quantities corresponding to the normalized density, parallel canonical momentum, parallel temperature and parallel heat flux, respectively. Clearly, because they refer to moments of the perturbation of the distribution function $\tilde{f}_{e}$, all of these fields have to be intended as fluctuations of the corresponding quantities.

From Eq. (23) it follows that one can obtain a hierarchy of evolution equations for the coefficients $g_{n}$ multiplying both sides of Eq. (1) times $H_{n} /\left(n_{0} \sqrt{n !}\right)$ and integrating over $v$. Making use of the recursive relation $H_{n+1}(x)=x H_{n}(x)-n H_{n-1}(x)$, the evolution equation for the generic coefficient $g_{n}$ reads [41]

$$
\begin{aligned}
& \frac{\partial g_{n}}{\partial t}=-\frac{c}{B}\left[\phi, g_{n}\right]+\sqrt{n+1} \frac{v_{t e}}{B}\left[A, g_{n+1}\right]+\sqrt{n} \frac{v_{t e}}{B}\left[A, g_{n-1}\right] \\
& -\sqrt{n+1} v_{t e} \frac{\partial}{\partial z} g_{n+1}-\sqrt{n} v_{t e} \frac{\partial}{\partial z} g_{n-1} \\
& +\delta_{n 1} v_{t e} \frac{\partial}{\partial z} \frac{e \phi}{T_{e}}-\sqrt{n !} v_{t e}^{2}\left(\delta_{n 0}+\delta_{n 2}\right) \frac{\partial}{\partial z} \frac{e A}{c T_{e}}
\end{aligned}
$$

It follows then that the evolution of $g_{n}$ is coupled to that of $g_{n-1}$ and to that of $g_{n+1}$, leading to an infinite hierarchy of fluid equations, as in the Vlasov case. Deriving a closed fluid model implies truncating this hierarchy and imposing a closure relation. In particular, a fluid model evolving the first $N+1$ coefficients, for a fixed positive integer $N \geq 3$, will consist of $N+1$ 
equations :

$$
\begin{aligned}
\frac{\partial g_{0}}{\partial t} & =-\frac{c}{B}\left[\phi, g_{0}\right]+\frac{v_{t e}}{B}\left[A, g_{1}\right]-v_{t e} \frac{\partial}{\partial z} g_{1}-v_{t e}^{2} \frac{\partial}{\partial z} \frac{e A}{c T_{e}} \\
\frac{\partial g_{1}}{\partial t} & =-\frac{c}{B}\left[\phi, g_{1}\right]+\sqrt{2} \frac{v_{t e}}{B}\left[A, g_{2}\right]+\frac{v_{t e}}{B}\left[A, g_{0}\right] \\
& -\sqrt{2} v_{t e} \frac{\partial}{\partial z} g_{2}-v_{t e} \frac{\partial}{\partial z} g_{0}+v_{t e} \frac{\partial}{\partial z} \frac{e \phi}{T_{e}} \\
\frac{\partial g_{2}}{\partial t} & =-\frac{c}{B}\left[\phi, g_{2}\right]+\sqrt{3} \frac{v_{t e}}{B}\left[A, g_{3}\right]+\sqrt{2} \frac{v_{t e}}{B}\left[A, g_{1}\right] \\
& -\sqrt{3} v_{t e} \frac{\partial}{\partial z} g_{3}-\sqrt{2} v_{t e} \frac{\partial}{\partial z} g_{1}-\sqrt{2} v_{t e}^{2} \frac{\partial}{\partial z} \frac{e A}{c T_{e}} \\
\frac{\partial g_{3}}{\partial t} & =-\frac{c}{B}\left[\phi, g_{3}\right]+2 \frac{v_{t e}}{B}\left[A, g_{4}\right]+\sqrt{3} \frac{v_{t e}}{B}\left[A, g_{2}\right] \\
& -2 v_{t e} \frac{\partial}{\partial z} g_{4}-\sqrt{3} v_{t e} \frac{\partial}{\partial z} g_{2} \\
& \vdots \\
\frac{\partial g_{N}}{\partial t} & =-\frac{c}{B}\left[\phi, g_{N}\right]+\sqrt{N+1} \frac{v_{t e}}{B}\left[A, \mathcal{L}\left(g_{0}, \cdots, g_{N}\right)\right]+\sqrt{N} \frac{v_{t e}}{B}\left[A, g_{N-1}(31)\right. \\
& -\sqrt{N+1} v_{t e} \frac{\partial}{\partial z} \mathcal{L}\left(g_{0}, \cdots, g_{N}\right)-\sqrt{N} v_{t e} \frac{\partial}{\partial z} g_{N-1}
\end{aligned}
$$

where in Eq. (31), the system has been closed imposing $g_{N+1}=\mathcal{L}\left(g_{0}, \cdots, g_{N}\right)$ where $\mathcal{L}$ is some operator acting on the first $N+1$ coefficients. We remark that above we have considered $N \geq 3$ because the first three equations of the hierarchy have a particular form which differs from that of Eq. (31) and because we wanted to emphasize the applications retaining a large number

of moments. However, the procedure described in the following applies also for $N=1$ and $N=2$, therefore, we can consider in general $N$ being any positive integer.

In the following we show that if one imposes the closure relation

$$
g_{N+1}=\alpha g_{N},
$$

where $\alpha$ is a constant, then the resulting fluid system possesses a Hamiltonian structure, as its parent model.

\section{Hamiltonian structure of the fluid model}

For an arbitrary positive integer $N$ we consider the fluid model describing the evolution of the $N+3$ fields $\left(\chi_{1}, \chi_{2}, \cdots, \chi_{N+3}\right)=\left(g_{0}, g_{1}, \cdots, g_{N}, n_{i}, D\right)$ 
obtained from the hybrid parent model. The fluid model consists of the $N+3$ evolution equations (4), (5), (27)-(31), where in Eq. $(31), \mathcal{L}\left(g_{0}, \cdots, g_{N}\right)$ is replaced by $\alpha g_{N}$. For the convenience of the reader we summarize the resulting fluid model:

$$
\begin{aligned}
\frac{\partial g_{0}}{\partial t} & =-\frac{c}{B}\left[\phi, g_{0}\right]+\frac{v_{t e}}{B}\left[A, g_{1}\right]-v_{t e} \frac{\partial}{\partial z} g_{1}-v_{t e}^{2} \frac{\partial}{\partial z} \frac{e A}{c T_{e}} \\
\frac{\partial g_{1}}{\partial t} & =-\frac{c}{B}\left[\phi, g_{1}\right]+\sqrt{2} \frac{v_{t e}}{B}\left[A, g_{2}\right]+\frac{v_{t e}}{B}\left[A, g_{0}\right] \\
& -\sqrt{2} v_{t e} \frac{\partial}{\partial z} g_{2}-v_{t e} \frac{\partial}{\partial z} g_{0}+v_{t e} \frac{\partial}{\partial z} \frac{e \phi}{T_{e}}, \\
& \vdots \\
\frac{\partial g_{N}}{\partial t} & =-\frac{c}{B}\left[\phi, g_{N}\right]+\alpha \sqrt{N+1} \frac{v_{t e}}{B}\left[A, g_{N}\right]+\sqrt{N} \frac{v_{t e}}{B}\left[A, g_{N-1}\right] \\
& -\alpha \sqrt{N+1} v_{t e} \frac{\partial}{\partial z} g_{N}-\sqrt{N} v_{t e} \frac{\partial}{\partial z} g_{N-1}, \\
\frac{\partial n_{i}}{\partial t} & =-\frac{c}{B}\left[\Gamma_{0}^{1 / 2} \phi, n_{i}\right]+\frac{n_{0}}{B}\left[\Gamma_{0}^{1 / 2} A, u_{i}\right]-n_{0} \frac{\partial u_{i}}{\partial z}, \\
\frac{\partial D}{\partial t} & =-\frac{c}{B}\left[\Gamma_{0}^{1 / 2} \phi, D\right]+\frac{T_{i}}{M B}\left[\Gamma_{0}^{1 / 2} A, n_{i}\right]-\frac{T_{i}}{M} \frac{\partial n_{i}}{\partial z}-\frac{e n_{0}}{M} \frac{\partial \Gamma_{0}^{1 / 2} \phi}{\partial z} .
\end{aligned}
$$

The model must be completed by the quasi-neutrality relation and Ampère's law which permit to determine $\phi$ and $A$. In the fluid model, of course these two relations have to be expressed in terms of fluid quantities. Making use of (23), it follows then that the quasi-neutrality relation and the Ampère's law for the fluid model read

$$
\begin{array}{r}
\frac{e^{2}}{T_{i}} n_{0}\left(1-\Gamma_{0}\right) \phi=-e \Gamma_{0}^{1 / 2} n_{i}+e n_{0} g_{0}, \\
\frac{c}{4 \pi} \Delta A-\frac{e^{2} n_{0}}{m_{e} c} A=-e n_{0} \Gamma_{0}^{1 / 2} u_{i}+e n_{0} v_{t e} g_{1} .
\end{array}
$$

respectively.

We remark that, moving to Fourier space, it is possible to show that Eqs. (38)-(39) can be cast in the form

$$
\begin{gathered}
\phi=L_{0}^{-1} e\left(\Gamma_{0}^{1 / 2} n_{i}-n_{0} g_{0}\right), \\
A=L_{1}^{-1} e\left(\Gamma_{0}^{1 / 2} D-n_{0} v_{t e} g_{1}\right),
\end{gathered}
$$


where $L_{0}$ and $L_{1}$ are linear invertible operators, which are symmetric with respect to the $L^{2}(\mathcal{D})$ inner product.

Showing that the system (33)-(37) possesses a Hamiltonian structure amounts to show, as above stated, that it can be cast in the form

$$
\frac{\partial \chi_{i}}{\partial t}=\left\{\chi_{i}, H\right\}, \quad i=1, \cdots, N+3,
$$

where $H=H\left(\chi_{1}, \cdots, \chi_{N+3}\right)=H\left(g_{0}, g_{1}, \cdots, g_{N}, n_{i}, D\right)$ is the Hamiltonian functional and $\{$,$\} is a Poisson bracket.$

As first step to construct the Hamiltonian structure of the model, we consider the evolution equations (36)-(37) for the ion variables. Because $\left\{n_{i}, \mathcal{H}_{e}\right\}_{i}=\left\{D, \mathcal{H}_{e}\right\}_{i}=0$ and given that $\left\{n_{i}, \mathcal{H}_{e}+\mathcal{H}_{c}+\mathcal{H}_{i}\right\}_{e}=\left\{D, \mathcal{H}_{e}+\right.$ $\left.\mathcal{H}_{c}+\mathcal{H}_{i}\right\}_{e}=0$, we obtain that, in the parent model, Eqs. (4)-(5), which are preserved as Eqs. (36)-(37) in the fluid model, can be written as

$$
\begin{aligned}
& \frac{\partial n_{i}}{\partial t}=\left\{n_{i}, \mathcal{H}_{c}+\mathcal{H}_{i}\right\}_{i}, \\
& \frac{\partial D}{\partial t}=\left\{D, \mathcal{H}_{c}+\mathcal{H}_{i}\right\}_{i},
\end{aligned}
$$

which is still a Hamiltonian form, given that $\{,\}_{i}$ is a Poisson bracket of its own. With regard to the Hamiltonian for the corresponding equations for the fluid model, we note that

$$
\begin{aligned}
& \mathcal{H}_{c}\left(g_{e}, n_{i}, D\right)=\tilde{\mathcal{H}}_{c}\left(g_{0}, g_{1}, n_{i}, D\right) \\
& =-\frac{e}{2} \int d^{3} x \phi\left(n_{0} g_{0}-\Gamma_{0}^{1 / 2} n_{i}\right)+\frac{e}{2 c} \int d^{3} x A\left(n_{0} v_{t e} g_{1}-\Gamma_{0}^{1 / 2} D\right),
\end{aligned}
$$

with $\phi$ and $A$ given by Eqs. (40)-(41). Therefore, the coupling term $\mathcal{H}_{c}$ in the Hamiltonian of the parent model can be exactly expressed, under the name $\tilde{\mathcal{H}}_{c}$, purely in terms of fluid quantities.

Because the bracket $\{,\}_{i}$ only depends on $n_{i}$ and $D$ and involves functional derivatives only with respect to $n_{i}$ and $D$, it follows that

$$
\begin{aligned}
& \frac{\partial n_{i}}{\partial t}=\left\{n_{i}, \tilde{\mathcal{H}}_{c}+\mathcal{H}_{i}\right\}_{i}, \\
& \frac{\partial D}{\partial t}=\left\{D, \tilde{\mathcal{H}}_{c}+\mathcal{H}_{i}\right\}_{i} .
\end{aligned}
$$

Eqs. (46)-(47) are then already written in Hamiltonian form with Hamiltonian functional and bracket only depending on fluid variables. The problem 
of determining the Hamiltonian structure of the model (33)-(37) reduces then to finding a functional $\mathcal{H}_{g}\left(g_{0}, \cdots, g_{N}\right)$ and a Poisson bracket $\{,\}_{g}$ only depending on $g_{0}, \cdots, g_{N}$ and not involving functionals derivatives with respect to $n_{i}$ and $D$, such that

$$
\begin{aligned}
\frac{\partial g_{0}}{\partial t}=\left\{g_{0}, \mathcal{H}_{g}+\tilde{\mathcal{H}}_{c}\right\}_{g} \\
\vdots \\
\frac{\partial g_{N}}{\partial t}=\left\{g_{N}, \mathcal{H}_{g}+\tilde{\mathcal{H}}_{c}\right\}_{g} .
\end{aligned}
$$

Once the functional $\mathcal{H}_{g}$ and the Poisson bracket $\{,\}_{g}$ are found, then the Hamiltonian structure for the total fluid model, as appearing in Eq. (42), will be given by a Hamiltonian functional

$$
H\left(g_{0}, \cdots, g_{N}, n_{i}, D\right)=\mathcal{H}_{g}+\tilde{\mathcal{H}}_{c}+\mathcal{H}_{i}
$$

and by a Poisson bracket $\{$,$\} with the following direct sum structure:$

$$
\{F, G\}=\{F, G\}_{g}+\{F, G\}_{i}
$$

The direct sum structure of the bracket of the parent model and the form of the coupling term in the Hamiltonian allow to treat separately the ion and the electron fluid equations. A similar procedure would of course be valid also if the parent model were fully drift-kinetic, provided that its Poisson bracket still had a direct sum structure separating the ion and electron dynamics.

We proceed first with determining a candidate functional for $\mathcal{H}_{g}$. It is natural, by analogy with what done previously for the ion equations, to try to derive the expression for $\mathcal{H}_{g}$ from the contribution to the Hamiltonian of the parent model only depending on the electrons, that is from $\mathcal{H}_{e}$. However, unlike $\mathcal{H}_{c}$ and $\mathcal{H}_{i}$, the functional $\mathcal{H}_{e}$ cannot be directly expressed in terms of the fluid variables. Therefore we consider, as in Ref. [12], the Hermite expansion (22) of $g_{e}$, but truncated at the first $N+1$ coefficients, which are those evolving in the fluid model. The expression for the truncated expansion reads

$$
g_{e}(x, y, z, v, t)=\sum_{n=0}^{N} \frac{g_{n}(x, y, z, t)}{\sqrt{n !}} H_{n}\left(\frac{v}{v_{t e}}\right) \mathcal{F}_{e}(v) .
$$

Replacing (52) in the expression for $\mathcal{H}_{e}$ and using the orthogonality relation 
(21) we obtain a functional

$$
\mathfrak{H}_{g}\left(g_{0}, \cdots, g_{N}\right)=\frac{n_{0} T_{e}}{2} \sum_{n=0}^{N} \int d^{3} x g_{n}^{2},
$$

which only depends on fluid electron variables. In order for $\mathfrak{H}_{g}$ to be a candidate for $\mathcal{H}_{g}$, it has to satisfy the property that $\tilde{\mathcal{H}}_{c}+\mathfrak{H}_{g}$ be the Hamiltonian for Eqs. (48)-(49). In particular, a necessary condition is that $\tilde{\mathcal{H}}_{c}+\mathfrak{H}_{g}$ has to be a conserved quantity for the system. In Ref. [12] it was shown that this is the case for $\alpha=0$. For $\alpha \neq 0$ it is easy to see that the only two additional contributions to $d\left(\tilde{\mathcal{H}}_{c}+\mathfrak{H}_{g}\right) / d t$ are given by

$$
\begin{gathered}
\alpha \sqrt{N+1} \frac{v_{t e}}{B} n_{0} T_{e} \int d^{3} x g_{N}\left[A, g_{N}\right]=0 \\
-\alpha \sqrt{N+1} n_{0} T_{e} v_{t e} \int d^{3} x g_{N} \frac{\partial g_{N}}{\partial z}=0
\end{gathered}
$$

where use has been made of integration by parts with vanishing boundary terms.

Consequently, $\tilde{\mathcal{H}}_{c}+\mathfrak{H}_{g}$ is a conserved quantity for Eqs. (48)-(49) and we can consider $\mathfrak{H}_{g}$ as a legitimate candidate for the functional $\mathcal{H}_{g}$.

As next step we remark that the generic equation for the system (33)-(35) can be written as

$$
\begin{aligned}
& \frac{\partial g_{m}}{\partial t}=-\frac{c}{B}\left[\phi, g_{m}\right]+\frac{v_{t e}}{B}\left[A, W_{m n} g_{n}\right]-v_{t e} \frac{\partial}{\partial z} W_{m n} g_{n}+\delta_{m 1} v_{t e} \frac{\partial}{\partial z} \frac{e \phi}{T_{e}} \\
& -\sqrt{m !}\left(\delta_{m 0}+\delta_{m 2}\right) v_{t e}^{2} \frac{\partial}{\partial z} \frac{e A}{c T_{e}}, \quad 0 \leq m \leq N, \quad
\end{aligned}
$$

where the sum over the repeated index $n$, with $0 \leq n \leq N$, is understood and where the elements of the matrix $W$ are given by

$$
W_{m n}=\sqrt{m} \delta_{m, n+1}+\sqrt{m+1} \delta_{m, n-1}+\alpha \sqrt{N+1} \delta_{m N} \delta_{n N}, \quad 0 \leq m \leq N, \quad 0 \leq n \leq N
$$


The matrix $W$ reads

$$
W=\left(\begin{array}{cccccc}
0 & 1 & 0 & 0 & \ldots & 0 \\
1 & 0 & \sqrt{2} & 0 & \ldots & 0 \\
0 & \sqrt{2} & 0 & \sqrt{3} & \ldots & 0 \\
0 & 0 & \sqrt{3} & 0 & \ldots & 0 \\
& \cdots & & & \ldots & \\
& \cdots & & & \ldots & \\
0 & 0 & 0 & \ldots & 0 & \sqrt{N} \\
0 & 0 & 0 & \ldots & \sqrt{N} & \alpha \sqrt{N+1}
\end{array}\right) .
$$

This real matrix is symmetric and consequently the eigenvalues $\lambda_{0}, \lambda_{1}, \cdots, \lambda_{N}$ of $W$ are real. Moreover there exists an orthogonal matrix $U$ such that $U^{T} W U=\Lambda$, where $\Lambda=\operatorname{diag}\left(\lambda_{0}, \lambda_{1}, \cdots, \lambda_{N}\right)$.

In order to unveil the Hamiltonian structure of the model, it turns out that it is most convenient to perform the change of variables $\left(g_{0}, g_{1}, \cdots, g_{N}\right) \rightarrow$ $\left(G_{0}, G_{1}, \cdots, G_{N}\right)$, where

$$
G_{i}=U_{i m}^{T} g_{m} .
$$

By applying the orthogonal transformation $U^{T}$ to Eq. (56), one obtains that the electron fluid equations in terms of the variables $G_{0}, G_{1}, \cdots, G_{N}$ read

$$
\begin{aligned}
& \frac{\partial G_{i}}{\partial t}=-\frac{c}{B}\left[\phi-\lambda_{i} \frac{v_{t e}}{c} A, G_{i}\right]-v_{t e} \lambda_{i} \frac{\partial G_{i}}{\partial z} \\
& +v_{t e} \sqrt{m !} U_{i m}^{T}\left(\delta_{m 1} \frac{\partial}{\partial z} \frac{e \phi}{T_{e}}-v_{t e}\left(\delta_{m 0}+\delta_{m 2}\right) \frac{\partial}{\partial z} \frac{e A}{c T_{e}}\right), \quad i=0, \cdots, N
\end{aligned}
$$

where use has been made of the property $U^{T} U=U U^{T}=I$. Note that in Eq. (60) the index $i$ is free and is not summed over.

From Eq. (60) it emerges that, in terms of the new variables, in the evolution equation for each field $G_{i}$, the coupling with the other fields occurs only through the presence of the potentials $\phi$ and $A$. Therefore, the orthogonal transformation $U^{T}$ removes the coupling that existed in the system formulated in terms of the old variables, where the evolution equation for each $g_{m}$ was explicitly dependent on $g_{m-1}$ and $g_{m+1}$ (apart for the equations (33) and (35)). In particular, in the 2D limit with translational invariance with respect to $z$, the model equations take the remarkable form

$$
\frac{\partial G_{i}}{\partial t}+\mathbf{v}_{i} \cdot \nabla G_{i}=0, \quad 0 \leq i \leq N .
$$


Each field $G_{i}$ is therefore a Lagrangian invariant advected by an incompressible velocity field

$$
\mathbf{v}_{i}=\frac{c}{B} \hat{z} \times \nabla\left(\phi-\lambda_{i} \frac{v_{t e}}{c} A\right) .
$$

The stream functions $(c / B)\left(\phi-\lambda_{i}\left(v_{t e} / c\right) A\right)$ associated with the velocity fields, are reminiscent of the stream function $(c / B)(\phi-(v / c) A)$ which, in the $2 \mathrm{D}$ limit, advects $g_{e}$ in the parent drift-kinetic equation (1), and which in turn originates from the $\mathbf{E} \times \mathbf{B}$ drift and from the stream of the guiding centers along the perpendicular component of the magnetic field. At the fluid level, these stream functions generate the velocity fields $\mathbf{v}_{i}$, which are indeed linear combinations of the $\mathbf{E} \times \mathbf{B}$ velocity and of the poloidal magnetic field. The coefficients of the linear combination depend on the eigenvalues $\lambda_{i}$ and consequently on the number of moments retained and on the value of $\alpha$. From examining the electron fluid equations (33)-(35) in the 2D limit for $\alpha=0$, it also follows that, if $N$ is even (so that the number of electron fluid equations is odd), then there always exists a Lagrangian invariant

$$
G_{L}=a\left(g_{0}+\sum_{n=1}^{N / 2}(-1)^{n} \sqrt{\frac{2 n-1}{2 n}} g_{2 n}\right),
$$

where $a$ is an arbitrary constant and $0 \leq L \leq N$. The Lagrangian invariant $G_{L}$ obeys the advection equation

$$
\frac{\partial G_{L}}{\partial t}=-\frac{c}{B}\left[\phi, G_{L}\right]
$$

Consequently, if $\alpha=0$ and if the hierarchy is truncated to an odd number of fluid moments, there is always a quantity which in 2D is advected purely by the $\mathbf{E} \times \mathbf{B}$ velocity. This corresponds to the matrix $W$ possessing a zero eigenvalue when odd-dimensional. The particular form taken by the system in the 2D limit in terms of the new variables, is suggestive of the structure of the Poisson bracket. Indeed, 2D models consisting of incompressible advection equations for Lagrangian invariants typically possess a Hamiltonian structure given by the direct sum of Lie-Poisson brackets analogous to the bracket for the 2D incompressible Euler equation [17, 42]. On the other hand, the extension of such Hamiltonian 2D models to 3D, according to the strong guide field ordering adopted in the " $\delta f$ approximation" at the drift-kinetic level, can be carried out by adopting the procedure described in Ref. [43]. 
On the basis of this procedure, from a 2D Poisson bracket of the form

$$
\{F, G\}_{\perp}=\sum_{i, j, k=0}^{N} W_{k}^{i j} \int d^{2} x \chi_{k}\left[\frac{\delta F}{\delta \chi_{i}}, \frac{\delta G}{\delta \chi_{j}}\right]
$$

with constant coefficients $W_{k}^{i j}$, one can generate a 3D Poisson bracket

$$
\{F, G\}=\sum_{i, j, k=0}^{N} W_{k}^{i j} \int d^{3} x \chi_{k}\left[\frac{\delta F}{\delta \chi_{i}}, \frac{\delta G}{\delta \chi_{j}}\right]+\sum_{m, n=0}^{N} b_{m n} \int d^{3} x \frac{\delta F}{\delta \chi_{m}} \frac{\partial}{\partial z} \frac{\delta G}{\delta \chi_{n}},
$$

provided that the constant coefficients $b_{m n}$ are such that $b_{m n}=b_{n m}$ and that $b_{r s} W_{r}^{i j}=b_{r i} W_{r}^{j s}=b_{r j} W_{r}^{s i}$. These two conditions ensure that the bracket (66) is antisymmetric and that it satisfies the Jacobi identity, respectively.

On the basis of these considerations, an ansatz for the form of the Poisson bracket of our system is provided by

$$
\{F, G\}=\sum_{i=0}^{N} a_{i} \int d^{3} x G_{i}\left[\frac{\delta F}{\delta G_{i}}, \frac{\delta G}{\delta G_{i}}\right]+\sum_{i, j=0}^{N} b_{i j} \int d^{3} x \frac{\delta F}{\delta G_{i}} \frac{\partial}{\partial z} \frac{\delta G}{\delta G_{j}},
$$

where $a_{0}, \cdots, a_{N}$ are constants and $b_{i j}$ are elements of a constant symmetric matrix. The first term in the right-hand side of Eq. (67) refers to the direct sum of Poisson brackets originated from 2D Euler-type brackets. The second contribution accounts for the 3D extension. Note that, because in this case $W_{k}^{i j}=a_{i} \delta_{i j} \delta_{j k}$, one sees that the condition for the Jacobi identity is automatically satisfied. Consequently, it is sufficient that the coefficients $b_{i j}$ be symmetric in their indices, in order for (67) to be a Poisson bracket.

In order to see whether the system (60) has a Hamiltonian structure with a Poisson bracket of the form (67), and a Hamiltonian corresponding to the candidate functional $\tilde{\mathcal{H}}_{c}+\mathfrak{H}_{g}$, one should verify that there exist coefficients $a_{i}$, with $i=0, \cdots, N$ and $b_{i j}$, with $i, j=0, \cdots, N$ such that the candidate Poisson bracket and Hamiltonian yield the model equations.

In order to verify this, it is necessary to transform also the candidate Hamiltonian $\tilde{\mathcal{H}}_{c}+\mathfrak{H}_{g}$ in terms of the new variables. Using $g_{n}=U_{n l} G_{l}$, from Eq. (45) and (53) we obtain

$$
\begin{array}{r}
\tilde{\mathcal{H}}_{c}\left(g_{0}, g_{1}, n_{i}, D\right)=\overline{\mathcal{H}}_{c}\left(G_{0}, G_{1}, \cdots, G_{N}, n_{i}, D\right) \\
=-\frac{e}{2} \int d^{3} x \phi\left(n_{0} U_{0 l} G_{l}-\Gamma_{0}^{1 / 2} n_{i}\right)+\frac{e}{2 c} \int d^{3} x A\left(n_{0} v_{t e} U_{1 l} G_{l}-\Gamma_{0}^{1 / 2} D\right), \\
\mathfrak{H}_{g}\left(g_{0}, \cdots, g_{N}\right)=\mathfrak{H}_{G}\left(G_{0}, \cdots, G_{N}\right)=\frac{n_{0} T_{e}}{2} \sum_{n=0}^{N} \int d^{3} x G_{n}^{2},
\end{array}
$$


where

$$
\begin{array}{r}
\phi=L_{0}^{-1} e\left(\Gamma_{0}^{1 / 2} n_{i}-n_{0} U_{0 l} G_{l}\right), \\
A=L_{1}^{-1} e\left(\Gamma_{0}^{1 / 2} D-n_{0} v_{t e} U_{1 l} G_{l}\right),
\end{array}
$$

From the inspection of the system determining the eigenvectors of $W$ we obtain that the first three rows of the matrix $U$ have the form

$$
U=\left(\begin{array}{ccccc}
v_{(0)} & v_{(1)} & \ldots & \ldots & v_{(N)} \\
\lambda_{0} v_{(0)} & \lambda_{1} v_{(1)} & \ldots & \ldots & \lambda_{N} v_{(N)} \\
\frac{\lambda_{0}^{2}-1}{\sqrt{2}} v_{(0)} & \frac{\lambda_{1}^{2}-1}{\sqrt{2}} v_{(1)} & \ldots & \ldots & \frac{\lambda_{N}^{2}-1}{\sqrt{2}} v_{(N)} \\
\cdot & \cdot & \ldots & \ldots & . \\
\cdot & \cdot & \ldots & \ldots & .
\end{array}\right)
$$

where $v_{(0)}, v_{(1)}, \cdots, v_{(N)}$ are constants determined by the condition of normalization on the eigenvectors.

By virtue of Eq. (72) we can rewrite Eqs. (68), (70) and (71) as

$$
\begin{array}{r}
\overline{\mathcal{H}}_{c}\left(G_{0}, G_{1}, \cdots, G_{N}, n_{i}, D\right)=-\frac{e}{2} \int d^{3} x \phi\left(n_{0} v_{(l)} G_{l}-\Gamma_{0}^{1 / 2} n_{i}\right) \\
+\frac{e}{2 c} \int d^{3} x A\left(n_{0} v_{t e} \lambda_{l} v_{(l)} G_{l}-\Gamma_{0}^{1 / 2} D\right), \\
\phi=L_{0}^{-1} e\left(\Gamma_{0}^{1 / 2} n_{i}-n_{0} v_{(l)} G_{l}\right), \\
A=L_{1}^{-1} e\left(\Gamma_{0}^{1 / 2} D-n_{0} v_{t e} \lambda_{l} v_{(l)} G_{l}\right) .
\end{array}
$$

From the expressions (69) and (73) one obtains that the functional derivatives of the candidate Hamiltonian are given by

$$
\frac{\delta}{\delta G_{i}}\left(\mathfrak{H}_{G}+\overline{\mathcal{H}}_{c}\right)=n_{0} T_{e} G_{i}-e n_{0} v_{(i)}\left(\phi-\lambda_{i} \frac{v_{t e}}{c} A\right), \quad i=0, \cdots, N
$$

The model equations (60), on the other hand, can be written as

$$
\begin{aligned}
& \frac{\partial G_{i}}{\partial t}=-\frac{c}{B}\left[\phi-\lambda_{i} \frac{v_{t e}}{c} A, G_{i}\right]-v_{t e} \lambda_{i} \frac{\partial G_{i}}{\partial z}+v_{t e} \lambda_{i} v_{(i)} \frac{\partial}{\partial z} \frac{e \phi}{T_{e}}-\frac{v_{t e}^{2}}{c} \lambda_{i}^{2} v_{(i)} \frac{\partial}{\partial z} \frac{e A}{T_{e}} \\
& i=0, \cdots, N
\end{aligned}
$$

Making use of the expression (76), one easily obtains that, by inserting $a_{i}=$ $c /\left(e B n_{0} v_{(i)}\right)$ and $b_{i j}=\delta_{i j} \lambda_{i} v_{t e} /\left(T_{e} n_{0}\right)$ in the expression for the ansatz of the 
Poisson bracket (67), the candidate Hamiltonian and Poisson bracket yield the following equations of motion:

$$
\begin{aligned}
& \frac{\partial G_{i}}{\partial t}=\left\{G_{i}, \mathfrak{H}_{G}+\overline{\mathcal{H}}_{c}\right\}=-\frac{c}{B}\left[\phi-\lambda_{i} \frac{v_{t e}}{c} A, G_{i}\right]-v_{t e} \lambda_{i} \frac{\partial G_{i}}{\partial z}+v_{t e} \lambda_{i} v_{(i)} \frac{\partial}{\partial z} \frac{e \phi}{T_{e}} \\
& -\frac{v_{t e}^{2}}{c} \lambda_{i}^{2} v_{(i)} \frac{\partial}{\partial z} \frac{e A}{T_{e}}, \quad i=0, \cdots, N .
\end{aligned}
$$

which correspond namely to the model equations (77). Consequently, we have shown that the Hamiltonian functional $\mathfrak{H}_{G}+\overline{\mathcal{H}}_{c}$ and the Poisson bracket

$$
\{F, G\}=\sum_{i=0}^{N} \frac{c}{e B n_{0} v_{(i)}} \int d^{3} x G_{i}\left[\frac{\delta F}{\delta G_{i}}, \frac{\delta G}{\delta G_{i}}\right]-\sum_{i=0}^{N} \frac{v_{t e}}{T_{e} n_{0}} \lambda_{i} \int d^{3} x \frac{\delta F}{\delta G_{i}} \frac{\partial}{\partial z} \frac{\delta G}{\delta G_{i}},
$$

provide the Hamiltonian structure for the system (77).

Making use of the relations

$$
G_{i}=U_{i m}^{T} g_{m}, \quad \frac{\delta \bar{F}}{\delta G_{i}}=U_{i m}^{T} \frac{\delta F}{\delta g_{m}},
$$

valid for any functional $F\left(g_{0}, \cdots, g_{N}\right)=\bar{F}\left(G_{0}, \cdots, G_{N}\right)$, one can then also express the Poisson bracket (79) in terms of the original variables. The result is

$$
\begin{aligned}
& \{F, G\}_{g}=\sum_{i, j, k, l=0}^{N} \frac{c}{e B n_{0} v_{(i)}} \int d^{3} x U_{i j}^{T} g_{j}\left[U_{i k}^{T} \frac{\delta F}{\delta g_{k}}, U_{i l}^{T} \frac{\delta G}{\delta g_{l}}\right] \\
& -\sum_{i, j, k=0}^{N} \frac{v_{t e}}{T_{e} n_{0}} \lambda_{i} \int d^{3} x U_{i j}^{T} \frac{\delta F}{\delta g_{j}} U_{i k}^{T} \frac{\partial}{\partial z} \frac{\delta G}{\delta g_{k}} .
\end{aligned}
$$

To summarize, we have shown that, for any positive integer $N$, the fluid model (33)-(37) possesses a Hamiltonian structure consisting of the Hamiltonian functional

$$
\begin{aligned}
& H=\mathcal{H}_{g}+\tilde{\mathcal{H}}_{c}+\mathcal{H}_{i} \\
& =\frac{n_{0} T_{e}}{2} \sum_{n=0}^{N} \int d^{3} x g_{n}^{2}-\frac{e}{2} \int d^{3} x \phi\left(n_{0} g_{0}-\Gamma_{0}^{1 / 2} n_{i}\right) \\
& +\frac{e}{2 c} \int d^{3} x A\left(n_{0} v_{t e} g_{1}-\Gamma_{0}^{1 / 2} D\right)+\frac{1}{2} \int d^{3} x\left(\frac{T_{i}}{n_{0}} n_{i}^{2}+\frac{M}{n_{0}} D^{2}\right),
\end{aligned}
$$


and of the Poisson bracket

$$
\begin{aligned}
& \{F, G\}=\{F, G\}_{g}+\{F, G\}_{i} \\
& =\sum_{i, j, k, l=0}^{N} \frac{c}{e B n_{0} v_{(i)}} \int d^{3} x U_{i j}^{T} g_{j}\left[U_{i k}^{T} \frac{\delta F}{\delta g_{k}}, U_{i l}^{T} \frac{\delta G}{\delta g_{l}}\right]-\sum_{i, j, k=0}^{N} \frac{v_{t e}}{T_{e} n_{0}} \lambda_{i} \int d^{3} x U_{i j}^{T} \frac{\delta F}{\delta g_{j}} U_{i k}^{T} \frac{\partial}{\partial z} \frac{\delta G}{\delta g_{k}} \\
& -\frac{c}{e B} \int d^{3} x\left(n_{i}\left[F_{n_{i}}, G_{n_{i}}\right]+D\left(\left[F_{n_{i}}, G_{D}\right]+\left[F_{D}, G_{n_{i}}\right]\right)+\frac{T_{i} n_{0}}{M} n_{i}\left[F_{D}, G_{D}\right]\right) \\
& -\frac{n_{0}}{M} \int d^{3} x\left(F_{D} \frac{\partial G_{n_{i}}}{\partial z}+F_{n_{i}} \frac{\partial G_{D}}{\partial z}\right),
\end{aligned}
$$

where $U$ is the orthogonal matrix that diagonalizes $W$, whereas $\lambda_{0}, \cdots, \lambda_{N}$ are the eigenvalues of $W$ and $v_{(0)}, \cdots, v_{(N)}$ are constants determined by the normalization condition on the orthonormal eigenvectors of $W$.

This shows that, by means of the closure relation (32), one can build reduced fluid models for an arbitrary number of fluid moments, such that the Hamiltonian character of the parent drift-kinetic model is respected. Dissipative terms can then of course be added, and the resulting model will then have a clear identification of a Hamiltonian core, with a total conserved energy (the Hamiltonian functional $H$ ) and the dissipative contributions.

The above result can also provide information about some models already presented in the literature. The hierarchy of the fluid equations (26) is indeed analogous to the one analyzed in Refs. [41, 14], although in the latter references, moments are taken with respect to a different generalized distribution function and a different coupling with the ion dynamics is adopted.

Truncating the hierarchy at $N=3$, which amounts to retain up to the heat flux dynamics, and considering the closure with $\alpha=0$, one unveils the Hamiltonian structure of simplified versions of the non-dissipative versions of the models present in Refs. [12, 7], in the limit where background inhomogeneities, gyroaverage effects and perpendicular moments are ignored.

Still setting $\alpha=0$ but considering only the dynamics of the first two moments, which amounts to an isothermal closure, one recovers the Hamiltonian model for magnetic reconnection of Ref. [44], in the absence of drifts. Restricting to the two equations for the electron dynamics, the Hamiltonian two-field model of Ref. [32] is recovered. 


\subsection{Casimir invariants}

One of the advantages of knowing the Hamiltonian structure of these models is that it allows to systematically identify further, often non trivial, invariants of motion, corresponding to the Casimirs of the Poisson bracket. In an infinite-dimensional case with noncanonical Poisson brackets, such as here, these are defined as the functionals $C$ such that

$$
\{C, F\}=0
$$

for all functional $F$ of the field variables. Because $d C / d t=0$, Casimir functionals impose further constraints on the dynamics, in addition to energy conservation.

Given the direct sum structure of the bracket (83), Casimirs of the system will be union of the sets of Casimirs of $\{,\}_{g}$ and $\{,\}_{i}$. Because Casimirs are solely a property of a Poisson bracket, they are best identified when the bracket is expressed in a simple form. In particular with regard to $\{,\}_{g}$, it is therefore convenient to transform it back to the form (79). It is then easy to see that the functionals

$$
C_{i}=\int d^{3} x G_{i}, \quad i=0, \cdots, N,
$$

are Casimirs of the system.

The Casimirs for $\{,\}_{i}$ had already been presented in Ref. [44] and we report them here:

$$
C_{ \pm}=\int d^{3} x I_{ \pm}, \quad \text { where } I_{ \pm}=D \pm \sqrt{\frac{T_{i}}{M}} n_{i}
$$

The model possesses then $N+3$ constants of motion, in addition to the total energy.

More interesting is the situation in the 2D limit. Indeed, assuming that $z$ be an ignorable coordinate, to each Casimir of the 3D case corresponds an infinite family of Casimirs. More precisely, the Casimirs for the 2D limit of the model are

$$
\begin{array}{r}
C_{i}=\int d^{2} x \mathcal{C}_{i}\left(G_{i}\right), \quad i=0, \cdots, N \\
C_{ \pm}=\int d^{2} x \mathcal{C}_{ \pm}\left(I_{ \pm}\right),
\end{array}
$$


where $\mathcal{C}_{0}, \cdots, \mathcal{C}_{N}, \mathcal{C}_{ \pm}$are arbitrary functions. Therefore, in the $2 \mathrm{D}$ limit, the evolution of the model is constrained by an infinite number of invariants. The presence of the $N+3$ infinite families of invariants reflects the fact that, as above noticed, in 2D the model can be cast in the form of evolution equations for Lagrangian invariants (as shown in Ref. [44], the ion equations (36) and (37) too can indeed be cast in such form, with $I_{ \pm}$being the Lagrangian invariants). The presence of these Lagrangian invariants, implies the existence of topological conservation laws. Indeed, contour lines of the Lagrangian invariants will be preserved during the dynamics and their reconnection will be forbidden. For two and four-field models, the role of such invariants for collisionless magnetic reconnection has been analyzed in Refs. [33, 34]. Given the incompressibility of the corresponding advecting velocity fields, the area enclosed by contour lines of the Lagrangian invariants will also be preserved.

Finally, we remark an analogy between the Casimirs of the Poisson bracket of the fluid model and those of the bracket of the parent model. Indeed, the Casimirs of the bracket (15) correspond to

$$
C=\int d^{3} x d v g_{e}, \quad C_{ \pm}=\int d^{3} x I_{ \pm}
$$

Assuming that $z$ be an ignorable coordinate, the Casimirs of the corresponding bracket become

$$
C=\int d^{2} x d v \mathcal{C}\left(g_{e}\right), \quad C_{ \pm}=\int d^{2} x \mathcal{C}_{ \pm}\left(I_{ \pm}\right)
$$

where $\mathcal{C}$ is an arbitrary function. Consequently, we see that all the Hamiltonian fluid models above constructed with the closure $g_{N+1}=\alpha g_{N}$ inherit from the parent hybrid model, the property of possessing a number of Casimirs equal to the number of dynamical variables. When the dependence on the $z$ coordinate is suppressed, each such Casimir generates an infinite family. In particular, the family of Casimirs $C$ of the parent model, reflects into the presence of the $N+1$ families of Casimirs $C_{0}, \cdots, C_{N}$ of Eq. (87). An analogy between the dynamics of Lagrangian invariants in a drift-kinetic model and in a two-field fluid model had been discussed in Refs. [45, 46, 47].

\subsection{Example: a 5+2 field Hamiltonian model}

In order to illustrate the above procedure, we derive a Hamiltonian fluid model evolving five moments for the electron fluid and two for the ion fluid, 
and we analyze its Hamiltonian structure. We consider the case $\alpha=0$, so that the closure amounts to setting $g_{5}=0$ in the hierarchy of equations. Consequently, from Eqs. (33)-(37), we obtain the model

$$
\begin{aligned}
\frac{\partial g_{0}}{\partial t} & =-\frac{c}{B}\left[\phi, g_{0}\right]+\frac{v_{t e}}{B}\left[A, g_{1}\right]-v_{t e} \frac{\partial}{\partial z} g_{1}-v_{t e}^{2} \frac{\partial}{\partial z} \frac{e A}{c T_{e}} \\
\frac{\partial g_{1}}{\partial t} & =-\frac{c}{B}\left[\phi, g_{1}\right]+\sqrt{2} \frac{v_{t e}}{B}\left[A, g_{2}\right]+\frac{v_{t e}}{B}\left[A, g_{0}\right] \\
& -\sqrt{2} v_{t e} \frac{\partial}{\partial z} g_{2}-v_{t e} \frac{\partial}{\partial z} g_{0}+v_{t e} \frac{\partial}{\partial z} \frac{e \phi}{T_{e}} \\
\frac{\partial g_{2}}{\partial t} & =-\frac{c}{B}\left[\phi, g_{2}\right]+\sqrt{3} \frac{v_{t e}}{B}\left[A, g_{3}\right]+\sqrt{2} \frac{v_{t e}}{B}\left[A, g_{1}\right] \\
& -\sqrt{3} v_{t e} \frac{\partial}{\partial z} g_{3}-\sqrt{2} v_{t e} \frac{\partial}{\partial z} g_{1}-\sqrt{2} v_{t e}^{2} \frac{\partial}{\partial z} \frac{e A}{c T_{e}}, \\
\frac{\partial g_{3}}{\partial t} & =-\frac{c}{B}\left[\phi, g_{3}\right]+2 \frac{v_{t e}}{B}\left[A, g_{4}\right]+\sqrt{3} \frac{v_{t e}}{B}\left[A, g_{2}\right] \\
& -2 v_{t e} \frac{\partial}{\partial z} g_{4}-\sqrt{3} v_{t e} \frac{\partial}{\partial z} g_{2}, \\
\frac{\partial g_{4}}{\partial t} & =-\frac{c}{B}\left[\phi, g_{4}\right]+2 \frac{v_{t e}}{B}\left[A, g_{3}\right]-2 v_{t e} \frac{\partial}{\partial z} g_{3}, \\
\frac{\partial n_{i}}{\partial t} & =-\frac{c}{B}\left[\Gamma_{0}^{1 / 2} \phi, n_{i}\right]+\frac{n_{0}}{B}\left[\Gamma_{0}^{1 / 2} A, u_{i}\right]-n_{0} \frac{\partial u_{i}}{\partial z}, \\
\frac{\partial D}{\partial t} & =-\frac{c}{B}\left[\Gamma_{0}^{1 / 2} \phi, D\right]+\frac{T_{i}}{M B}\left[\Gamma_{0}^{1 / 2} A, n_{i}\right]-\frac{T_{i}}{M} \frac{\partial n_{i}}{\partial z}-\frac{e n_{0}}{M} \frac{\partial \Gamma_{0}^{1 / 2} \phi}{\partial z}
\end{aligned}
$$

From Eq. (82) one obtains immediately that the Hamiltonian of the model is given by

$$
\begin{aligned}
& H=\frac{n_{0} T_{e}}{2} \sum_{n=0}^{4} \int d^{3} x g_{n}^{2}-\frac{e}{2} \int d^{3} x \phi\left(n_{0} g_{0}-\Gamma_{0}^{1 / 2} n_{i}\right) \\
& +\frac{e}{2 c} \int d^{3} x A\left(n_{0} v_{t e} g_{1}-\Gamma_{0}^{1 / 2} D\right)+\frac{1}{2} \int d^{3} x\left(\frac{T_{i}}{n_{0}} n_{i}^{2}+\frac{M}{n_{0}} D^{2}\right),
\end{aligned}
$$

With regard to the Poisson bracket, we know from Eq. (83) that it is of the form $\{F, G\}=\{F, G\}_{g}+\{F, G\}_{i}$, with $\{F, G\}_{i}$ given by Eq. (17). We then just need to determine $\{F, G\}_{g}$. With this purpose, we first notice that the 
matrix $W$ in this case reads

$$
W=\left(\begin{array}{ccccc}
0 & 1 & 0 & 0 & 0 \\
1 & 0 & \sqrt{2} & 0 & 0 \\
0 & \sqrt{2} & 0 & \sqrt{3} & 0 \\
0 & 0 & \sqrt{3} & 0 & 2 \\
0 & 0 & 0 & 2 & 0
\end{array}\right)
$$

and that its eigenvalues are given by

$$
\begin{array}{ll}
\lambda_{0}=-\sqrt{5+\sqrt{10}}, & \lambda_{1}=\sqrt{5+\sqrt{10}}, \quad \lambda_{2}=-\sqrt{5-\sqrt{10}} \\
\lambda_{3}=\sqrt{5-\sqrt{10}}, & \lambda_{4}=0 .
\end{array}
$$

According to the above introduced prescription, there exists for this model a change of variables $\left(g_{0}, \cdots, g_{4}\right) \rightarrow\left(G_{0}, \cdots, G_{4}\right)$ in terms of which the Poisson bracket $\{F, G\}_{g}$ takes a simple direct sum structure. The new variables are given by

$$
G_{i}=U_{i l}^{T} g_{l}, \quad i=0, \cdots, 4
$$

where

$$
\begin{aligned}
& U= \\
& \left(\begin{array}{ccccc}
-\frac{\sqrt{2}-\sqrt{5}}{2 \sqrt{15}} & -\frac{\sqrt{2}-\sqrt{5}}{2 \sqrt{15}} & -\frac{\sqrt{2}+\sqrt{5}}{2 \sqrt{15}} & -\frac{\sqrt{2}+\sqrt{5}}{2 \sqrt{15}} & \frac{2 \sqrt{2}}{\sqrt{15}} \\
\frac{\sqrt{5+\sqrt{10}}(\sqrt{2}-\sqrt{5})}{2 \sqrt{15}} & -\frac{\sqrt{5+\sqrt{10}}(\sqrt{2}-\sqrt{5})}{2 \sqrt{15}} & \frac{\sqrt{5-\sqrt{10}}(\sqrt{2}+\sqrt{5})}{2 \sqrt{15}} & -\frac{\sqrt{5-\sqrt{10}}(\sqrt{2}+\sqrt{5})}{2 \sqrt{15}} & 0 \\
\frac{1+\sqrt{10}}{2 \sqrt{15}} & \frac{1+\sqrt{10}}{2 \sqrt{15}} & \frac{1-\sqrt{10}}{2 \sqrt{15}} & \frac{1-\sqrt{10}}{2 \sqrt{15}} & -\frac{2}{\sqrt{15}} \\
-\frac{\sqrt{5+\sqrt{10}}}{2 \sqrt{5}} & \frac{\sqrt{5+\sqrt{10}}}{2 \sqrt{5}} & -\frac{\sqrt{5-\sqrt{10}}}{2 \sqrt{5}} & \frac{\sqrt{5-\sqrt{10}}}{2 \sqrt{5}} & 0 \\
\frac{1}{\sqrt{5}} & \frac{1}{\sqrt{5}} & \frac{1}{\sqrt{5}} & \frac{1}{\sqrt{5}} & \frac{1}{\sqrt{5}}
\end{array}\right),
\end{aligned}
$$

is the orthogonal matrix which diagonalizes $W$.

We can then express the Hamiltonian structure of the model in terms of 
the new variables. The Hamiltonian (98) transforms into

$$
\begin{aligned}
& H=\frac{n_{0} T_{e}}{2} \sum_{n=0}^{4} \int d^{3} x G_{n}^{2} \\
& +\frac{e n_{0}}{2} \int d^{3} x \phi\left(\frac{\sqrt{2}-\sqrt{5}}{2 \sqrt{15}} G_{0}+\frac{\sqrt{2}-\sqrt{5}}{2 \sqrt{15}} G_{1}+\frac{\sqrt{2}+\sqrt{5}}{2 \sqrt{15}} G_{2}+\frac{\sqrt{2}+\sqrt{5}}{2 \sqrt{15}} G_{3}\right. \\
& \left.-\frac{2 \sqrt{2}}{\sqrt{15}} G_{4}+\Gamma_{0}^{1 / 2} \frac{n_{i}}{n_{0}}\right) \\
& -\frac{e n_{0} v_{t e}}{2 c} \int d^{3} x A\left(\frac{\sqrt{5+\sqrt{10}}(\sqrt{2}-\sqrt{5})}{2 \sqrt{15}} G_{0}+\frac{\sqrt{5+\sqrt{10}}(\sqrt{2}-\sqrt{5})}{2 \sqrt{15}} G_{1}\right. \\
& \left.-\frac{\sqrt{5-\sqrt{10}}(\sqrt{2}+\sqrt{5})}{2 \sqrt{15}} G_{2}+\frac{\sqrt{5-\sqrt{10}}(\sqrt{2}+\sqrt{5})}{2 \sqrt{15}} G_{3}+\frac{\Gamma_{0}^{1 / 2} D}{n_{0} v_{t e}}\right) \\
& +\frac{1}{2} \int d^{3} x\left(\frac{T_{i}}{n_{0}} n_{i}^{2}+\frac{M}{n_{0}} D^{2}\right) .
\end{aligned}
$$

The Poisson bracket, on the other hand, can be written as

$$
\begin{aligned}
& \{F, G\}=\frac{c}{e B n_{0}} \int d^{3} x\left(-2 \frac{\sqrt{15}}{\sqrt{2}-\sqrt{5}} G_{0}\left[F_{G_{0}}, G_{G_{0}}\right]-2 \frac{\sqrt{15}}{\sqrt{2}-\sqrt{5}} G_{1}\left[F_{G_{1}}, G_{G_{1}}\right]\right. \\
& \left.-2 \frac{\sqrt{15}}{\sqrt{2}+\sqrt{5}} G_{2}\left[F_{G_{2}}, G_{G_{2}}\right]-2 \frac{\sqrt{15}}{\sqrt{2}+\sqrt{5}} G_{3}\left[F_{G_{3}}, G_{G_{3}}\right]+\frac{\sqrt{15}}{2 \sqrt{2}} G_{4}\left[F_{G_{4}}, G_{G_{4}}\right]\right) \\
& -\sum_{i=0}^{4} \frac{v_{t e}}{T_{e} n_{0}} \lambda_{i} \int d^{3} x F_{G_{i}} \frac{\partial}{\partial z} G_{G_{i}} \\
& -\frac{c}{e B} \int d^{3} x\left(n_{i}\left[F_{n_{i}}, G_{n_{i}}\right]+D\left(\left[F_{n_{i}}, G_{D}\right]+\left[F_{D}, G_{n_{i}}\right]\right)+\frac{T_{i} n_{0}}{M} n_{i}\left[F_{D}, G_{D}\right]\right) \\
& -\frac{n_{0}}{M} \int d^{3} x\left(F_{D} \frac{\partial G_{n_{i}}}{\partial z}+F_{n_{i}} \frac{\partial G_{D}}{\partial z}\right),
\end{aligned}
$$

and making use of the transformation rules (80), it can also be expressed in terms of the original variables of the model.

From the considerations of Sec. 4.1 we can deduce that the model possesses seven Casimir invariants corresponding to $\int d^{3} x I_{ \pm}, \int d^{3} x G_{i}$, with $i=$ 
$0, \cdots, 4$. In the $2 \mathrm{D}$ limit, the model can be formulated as a system of seven advection equations for Lagrangian invariants. In particular, if we consider the five Lagrangian invariants $G_{0}, \cdots, G_{4}$ associated with the electron fluid moments, we see that these are advected by incompressible flows with stream functions:

$$
\begin{array}{rlrl}
\phi_{0}=\frac{c}{B}\left(\phi+\sqrt{5+\sqrt{10}} \frac{v_{t e}}{c} A\right), & \phi_{1}=\frac{c}{B}\left(\phi-\sqrt{5+\sqrt{10}} \frac{v_{t e}}{c} A\right), \\
\phi_{2}=\frac{c}{B}\left(\phi+\sqrt{5-\sqrt{10}} \frac{v_{t e}}{c} A\right), & \phi_{3}=\frac{c}{B}\left(\phi-\sqrt{5-\sqrt{10}} \frac{v_{t e}}{c} A\right), \\
\phi_{4}=\frac{c}{B} \phi .
\end{array}
$$

The advecting flows are then organized in such a way that in each pair $\left(\phi_{0}, \phi_{1}\right)$ and $\left(\phi_{2}, \phi_{3}\right)$, the contribution to the velocity due to the poloidal magnetic field, in one case adds to the $\mathbf{E} \times \mathbf{B}$ flow and in the other case it subtracts to it. In the two-moment models of Ref. [33], a similar feature was present and led to clockwise and anticlockwise rotation of the Lagrangian invariants. As anticipated in Sec. 4, given that in this case $\alpha=0$ and $N$ is even, we also have a Lagrangian invariant, corresponding to $G_{L}=G_{4}=$ $(2 \sqrt{2} / \sqrt{15}) g_{0}-(2 / \sqrt{15}) g_{2}+(1 / \sqrt{15}) g_{4}$, which is advected purely by the $\mathbf{E} \times \mathbf{B}$ flow, associated with the zero eigenvalue $\lambda_{4}$.

We remark that a mechanical analog of fluid models obtained by taking moments of Hermite polynomials, was proposed in Ref. [3]. According to such analogy, the hierarchy of fluid equations can be put in correspondence with the equations for an infinite system of masses connected by springs, each mass being subject to an elastic potential exerted by the neighboring masses. Imposing the closure $g_{N+1}=0$, as in the model under consideration, corresponds to replacing one of the masses by a fixed wall. Consistently with our result, in Ref. [3] it is pointed out that such closure leads to an energyconserving system. Here we have shown that, in addition to be energyconserving, the system, in terms of fluid Eulerian variables, possesses a nontrivial noncanonical Hamiltonian structure.

\section{Discussion and conclusions}

We have shown that it is possible to construct Hamiltonian electromagnetic reduced fluid models of an arbitrary number $N+1$ of moments by imposing the closure $g_{N+1}=\alpha g_{N}$ to the hierarchy of equations obtained 
from a Hamiltonian drift-kinetic system in the " $\delta f$ approximation". This closure therefore, respects the Hamiltonian nature of the parent model. We have treated the case in which the parent model is actually a hybrid model coupling a drift-kinetic description for the electrons and a gyrofluid description for the ions, but the result can easily be extended to the case of a purely drift-kinetic Hamiltonian parent model, given that the direct sum structure of the corresponding Poisson bracket allows to treat separately the moment equations of the different species.

A first immediate consequence of this result is that, for the fluid models derived with this procedure, a conserved total energy is automatically available. This energy is derived self-consistently from the Hamiltonian of the parent model and corresponds to the conserved total energy derived in Ref. [12] for the $\alpha=0$ case.

The knowledge of the Hamiltonian structure, in addition to providing the correct definition of the total energy, corresponding to the Hamiltonian of the system, offers further information. In particular, Hamiltonian systems with noncanonical Poisson brackets, such as the fluid models derived here, typically possess further conserved quantities denoted as Casimir invariants.

We have identified a change of variables $\left(g_{0}, \cdots, g_{N}\right) \rightarrow\left(G_{0}, \cdots, G_{N}\right)$ which transforms the original set of fluid variables to a new set of variables which has first suggested the form of the Poisson bracket and in terms of which the Poisson bracket takes a simple direct sum structure. This helped to identify the existence of $N+3$ Casimir invariants $(\mathrm{N}+1$ associated with the electron fluid and two related to the ion fluid) in the general case, and in particular, the existence of $N+3$ infinite families of Casimir invariants which constrain the dynamics of the system in the 2D limit. Interestingly, in the 2D limit, the model can be reformulated as a system of $N+3$ advection equations for Lagrangian invariants. The Lagrangian invariants correspond to the new variables $G_{0}, \cdots, G_{N}$ for the electron fluid and to the already known invariants $I_{ \pm}$for the ion fluid. These invariants are advected by incompressible flows which are linear combinations of the $\mathbf{E} \times \mathbf{B}$ velocity and of a velocity proportional to the poloidal magnetic field. These are reminiscent of the velocities appearing in the equations of motion for the guiding centers. The coefficients of the linear combinations depend on the eigenvalues of the symmetric matrix $W$, and thus they depend on the number of fluid moments retained and on the value of $\alpha$ in the closure relation. We have remarked that an example of such structure in terms of Lagrangian invariants had been found for two-field and four-field Hamiltonian models for magnetic reconnec- 
tion in Refs. [32] and [44], respectively. These two models indeed turn out to be particular cases of the family of Hamiltonian fluid models studied in this paper, and they correspond to retaining only the first two moments of the electron and ion fluid hierarchies and to setting $\alpha=0$. Our result shows then that the possibility of reexpressing the model as a system of advection equations for Lagrangian invariants is not a peculiar property of the models of Refs. [32, 44] but is a generic feature of $2 \mathrm{D}$ fluid models derived from drift-kinetic equations of the form (1), with the closure $g_{N+1}=\alpha g_{N}$, and can therefore be extended to an arbitrary number of moments. One could then speculate that the phase mixing process of the Lagrangian invariants of the models of Refs. [32, 44], which has been shown to lead to the generation of small scales in fields such as vorticity and current density [33, 34], could be generalized to more refined fluid models with higher order closures involving for instance pressure and heat fluxes.

The knowledge of the Casimirs also leads to two natural applications, which will be the subject of forthcoming publications. On one hand, one could take advantage of them in order to derive energy stability conditions by means of the Energy-Casimir method [18, 17]. This method could be particularly fruitful in the 2D case, where infinite families of Casimirs are present. On the other hand, it would be natural to investigate numerically the robustness of some of the Casimir invariants (for instance the quadratic ones) and of the Hamiltonian in the presence of dissipation. This might suggest an hypothesis of selective decay for these reduced models.

As the number of moments $N+1$ increases, however, determining analytically eigenvalues and eigenvectors of $W$, which are used to construct the Poisson bracket, can become challenging. On the other hand, because we have proved the existence of the Hamiltonian structure for any $N$ and because the Hamiltonian functional (82) is already available, one can proceed to determine the perpendicular Poisson bracket $\{,\}_{\perp}$ of Eq. (65), for any fixed $N$, using the procedure adopted for instance in Refs. [48, 49]. This procedure relies on the knowledge of the Hamiltonian functional and on a commutation condition on the tensors $W_{k}^{i j}$ of Eq. (65), which ensures the Jacobi identity. The extension of the bracket to 3D can then be carried out according to the procedure described in Ref. [43] and recalled in Sec. 4.

In view of the presented results, a natural question to investigate is about whether there exist other closures that could lead to a Hamiltonian structure. With regard to this issue, at the moment, we can state the following. The structure of the model equations suggests that, in the presence of a Hamilto- 
nian with only quadratic terms, Poisson brackets of the form (65), obtained by extension of a Lie-Poisson bracket [42], could provide the form of the bracket in the $2 \mathrm{D}$ limit, in the case of general linear closure relations of the form $g_{N+1}=\alpha_{i} g_{i}$, with $i=0, \cdots, N$. However, the Hamiltonian functional (82), which is the one obtained self-consistently from the Hamiltonian of the parent model, would not be conserved for closures other than the one treated in this paper. Therefore, one should at least figure out a different candidate for the Hamiltonian. Moreover, we notice that, also the identification of a set of normal fields $G_{0}, \cdots, G_{N}$ with our procedure cannot be extended in the presence of a general linear closure. Indeed, the identification of such normal fields essentially relies on the symmetry of the matrix $W$. A linear closure involving moments of order lower than $N$ would spoil the symmetry of such matrix.

Another natural question to explore concerns the physical meaning of the parameter $\alpha$. As above noticed, models corresponding to $\alpha=0$ are already present in the literature $[12,7,44,32]$ but, to the best of our knowledge, the closure with $\alpha \neq 0$ still has not been adopted. In order to get a first insight about the physical consequences of such a closure, we can consider a very simple example. By neglecting ion density and velocities in Eqs. (38) and (39), which is consistent for instance with the ordering applied in Ref. [41], and considering the limit $k_{\perp}^{2} \rho_{i}^{2} \ll 1$, the quasi-neutrality relation and Ampère's law become

$$
\begin{array}{r}
\frac{M c^{2}}{B^{2}} \Delta \phi=e g_{0}, \\
\frac{c}{4 \pi} \Delta A-\frac{e^{2} n_{0}}{m_{e} c} A=e n_{0} v_{t e} g_{1} .
\end{array}
$$

The Hamiltonian character is not altered because the form of Eqs. (104)(105) still permits to express $\phi$ and $A$ in the form (9). The set of electron fluid equations (33)-(35), complemented by Eqs. (104)-(105) becomes then a closed Hamiltonian system. We consider then $N=1$ and the $2 \mathrm{D}$ limit. The 
resulting system can be written as

$$
\begin{array}{r}
\frac{\partial \Delta \phi}{\partial t}+\frac{c}{B}[\phi, \Delta \phi]-\frac{B}{4 \pi M c n_{0}}[A, \Delta A]=0 \\
\frac{\partial}{\partial t}\left(A-\frac{m_{e} c^{2}}{4 \pi e^{2} n_{0}} \Delta A\right)+\frac{c}{B}\left[\phi, A-\frac{m_{e} c^{2}}{4 \pi e^{2} n_{0}} \Delta A\right]+\frac{m_{e} M c^{3} v_{t e}^{3}}{e^{2} B^{3}}[A, \Delta \phi] \\
+\alpha \sqrt{2} \frac{m_{e} c^{2} v_{t e}}{4 \pi e^{2} n_{0} B}[A, \Delta A]=0 .
\end{array}
$$

This corresponds to the two-field model investigated in Refs. [32, 33], modified by the additional term depending on $\alpha$. We linearize then the system (106)-(107) about the equilibrium $A_{\text {eq }}=\mathcal{B} x, \phi_{\text {eq }}=0$, with constant $\mathcal{B}$, and which corresponds to a homogeneous magnetic field in the "poloidal" plane, with no $\mathbf{E} \times \mathbf{B}$ flow. Upon considering perturbations of the form $\tilde{\phi}(x, y, t)=$ $\sum_{\mathbf{k} \in \mathbb{Z}^{2} \backslash\{(0,0)\}} \phi_{\mathbf{k}} \exp (i(\mathbf{k} \cdot \mathbf{x}-\omega t)), \tilde{A}(x, y, t)=\sum_{\mathbf{k} \in \mathbb{Z}^{2}} A_{\mathbf{k}} \exp (i(\mathbf{k} \cdot \mathbf{x}-\omega t))$ (the $k_{x}=k_{y}=0$ mode has been removed from the potential fluctuations in order for the inverse operator $L_{0}^{-1}$ to be defined) we obtain that the resulting dispersion relation reads

$$
\bar{\omega}=\frac{-\bar{\alpha} \pm \sqrt{\bar{\alpha}^{2}+4\left(1+k_{\perp}^{2} \rho_{s}^{2}\right)\left(1+d_{e}^{2} k_{\perp}^{2}\right)}}{2\left(1+d_{e}^{2} k_{\perp}^{2}\right)}
$$

where

$$
\begin{array}{r}
\bar{\omega}=\frac{\omega}{k_{y}} \frac{\sqrt{4 \pi M n_{0}}}{\mathcal{B}}, \quad \bar{\alpha}=\alpha \sqrt{2} d_{e}^{2} k_{\perp}^{2} \frac{\sqrt{4 \pi M n_{0}}}{B} v_{t e} \\
\rho_{s}=\sqrt{\frac{T_{e}}{T_{i}}} \rho_{i}, \quad d_{e}=c \sqrt{\frac{m_{e}}{4 \pi e^{2} n_{0}}} .
\end{array}
$$

From (108), in the limit $\alpha=0$, one recovers the usual dispersion relation for kinetic Alfvén waves accounting for electron inertia. We see, on the other hand, that if $\alpha \neq 0$ the two branches of kinetic Alfvén waves get modified. No instability is introduced by the term depending on $\alpha$. However, the frequency of the modes gets altered. For instance, for $\alpha>0$, one can deduce from Eq. (108) that, for each $\mathbf{k}$ the frequencies of the two branches are lower with respect to the corresponding branches of kinetic Alfvén waves.

From a more practical point of view, one could also try to investigate whether, for a given fluid model, some optimal choice of the parameter $\alpha$ 
exists, such that some features (e.g. linear growth rates) of the parent driftkinetic model can be approximated at best.

Finally, we would like to point out the obvious limitations which our analysis still suffers from. A main one concerns the relative simplicity of the parent drift-kinetic equation. Indeed, it would of course be important to extend the analysis to include the dependence of the distribution function on the magnetic moment. This could lead to the investigation of fluid models accounting for moment anisotropies between the directions parallel and perpendicular to the guide field. Also, further drifts, such as those associated with background magnetic gradients or curvature, which can be important for instance in magnetic fusion applications, should be incorporated. The extension to gyrokinetic systems as parent models is also desirable and, together with the above mentioned generalizations, it is part of on-going work on the subject.

\section{Acknowledgments}

The author acknowledges useful discussions with the Nonlinear Dynamics Team of the Centre de Physique Théorique, with Alain Brizard and with Claudio di Troia. Financial support was received from the Agence Nationale de la Recherche (ANR GYPSI n. 2010 BLAN 941 03) and from the CNRS through the PEPS project GEOPLASMA2.

\section{References}

[1] G.W. Hammett, F.W. Perkins, Phys. Rev. Lett. 64, (1990) 3019.

[2] G.W. Hammett, W. Dorland, F. W. Perkins, Phys. Fluids B 4, (1992) 2052.

[3] G.W. Hammett, M.A. Beer, W. Dorland, S.C. Cowley, S.A. Smith, Plasma Phys. Control. Fusion 35, (1993) 973.

[4] W. Dorland, G.W. Hammett, Phys. Fluids B 5, (1993) 812.

[5] M.A. Beer, G. Hammett, Phys. Plasmas 3, (1996) 4046.

[6] P.B. Snyder, G.W. Hammett, W. Dorland, Phys. Plasmas 4, (1997) 3974. 
[7] P.B. Snyder, G.W. Hammett, Phys. Plasmas 8, (2001) 3199.

[8] T. Passot, P.L. Sulem, Phys. Plasmas 11, (2004) 5173.

[9] T. Passot, P.L. Sulem, Phys. Plasmas 14, (2007) 082502.

[10] T. Passot, P.L. Sulem, P. Hunana, Phys. Plasmas 19, (2012) 082113.

[11] A. Brizard, Phys. Fluids B 4, (1992) 1213.

[12] B. Scott, Phys. Plasmas 17, (2010) 102306.

[13] H. Sugama, T.-H Watanabe, W. Horton, Phys. Plasmas 8, (2001) 2617.

[14] N.F. Loureiro, A.A. Schekochihin, A. Zocco, Phys. Rev. Lett. 111, (2013) 025002 .

[15] P. J. Morrison, Phys. Lett. 80A, (1980) 383.

[16] J. E. Marsden, A. Weinstein, Physica D 4, (1982) 394.

[17] P.J. Morrison, Rev. Mod. Phys. 70 (1998) 467.

[18] D.D. Holm, J.E. Marsden, T. Ratiu, A. Weinstein, Phys. Reports 123, (1985) 1.

[19] V. Zeitlin, in Nonlinear World edited by V. Baryakhtar et al. (World Scientific, Singapore) 1, (1989) 717.

[20] V. Zeitlin, Phys. Lett. A 339, (2005) 316.

[21] J. Gibbons, Physica D 3 (1981) 503.

[22] B.A. Kupershmidt, Ju. I Manin, Funktsional. Anal. i Prilozhen. 1, (1978) 25.

[23] J. Gibbons, D.D. Holm, C. Tronci, Phys. Lett. A 372, (2008) 1024.

[24] J. Gibbons, D.D. Holm, C. Tronci, Phys. Lett. A 372, (2008) 4184.

[25] J.E. Marsden, P.J. Morrison, A. Weinstein, Contemp. Math. 28, (1984) 115.

[26] A. A. Chesnokov, M.V. Pavlov, Acta Appl. Math. 122 (2012) 367. 
[27] L. de Guillebon, C. Chandre, Phys. Lett. A 376, (2012) 3172.

[28] E. Tassi, J. Phys. A: Math. Theor. 47, (2014) 195501.

[29] E. Tassi, Eur. Phys. J. D 68, (2014) 196.

[30] E. Tassi, Journal of Physics: Conference Series 561, (2014) 012018.

[31] M. Perin, C. Chandre, P.J. Morrison, E. Tassi, Annals of Physics 348, (2014) 50.

[32] E. Cafaro, D. Grasso, F. Pegoraro, F. Porcelli, A. Saluzzi, Phys. Rev. Lett. 80, (1998) 4430.

[33] D. Grasso, F. Califano, F. Pegoraro, F. Porcelli, Phys. Rev. Lett. 86, (2001) 5051.

[34] L. Comisso, D. Grasso, E. Tassi, F.L. Waelbroeck, Phys. Plasmas 19, (2012) 042103.

[35] H.J. de Blank, Phys. Plasmas 8, (2001) 3927.

[36] H.J. de Blank, Proceedings of the 28th Conference on Contr. Fusion and Plasma Phys., Funchal, 18-22 June 2001, Ed. ECA 25A, (2001) 1709.

[37] F.L. Waelbroeck, R.D. Hazeltine, P.J. Morrison, Phys. Plasmas 16, (2009) 032109.

[38] F.C. Grant, M.R. Feix, Phys. Fluids 10, (1967) 1356.

[39] T.P. Armstrong, R.C. Harding, G. Knorr, D. Montgomery, Methods in Computational Physics 9 (1970) 29.

[40] W.L. Sadowski, NASA SP-153: Symposium on Computer Simulation of Plasma and Many-Body Problems, Clearinghouse for Federal Scientific Information, Springfield, VA, USA (1967) 433.

[41] A. Zocco, A.A. Schekochihin, Phys. Plasmas 18, (2011) 102309.

[42] J.-L. Thiffeault, P.J. Morrison, Physica D 136 (2000) 205.

[43] E. Tassi, P.J. Morrison, D. Grasso, F. Pegoraro, Nucl. Fusion 50, (2010) 034007. 
[44] F.L. Waelbroeck, E. Tassi, Commun. Nonlinear Sci. Numer. Simulat. $17,(2012) 2171$.

[45] T.V. Liseikina, F. Pegoraro and E. Yu. Echkina, Phys. Plasmas 11, (2004) 3535.

[46] F. Pegoraro, T. Liseikina and E. Yu. Echkina, Transport Theory and Statistical Physics 34, (2005) 243.

[47] F. Pegoraro, T. Liseikina and E. Yu. Echkina, Phys. Scripta T116, (2005) 88.

[48] F.L. Waelbroeck, P.J. Morrison, W. Horton, Plasma Phys. and Contr. Fusion 46, (2004) 1331.

[49] E. Tassi, P.J. Morrison, F.L. Waelbroeck, D. Grasso, Plasma Phys. and Contr. Fusion 50, (2008) 085014. 